\title{
Quantification of the influence of drugs on zebrafish larvae swimming kinematics and energetics
}

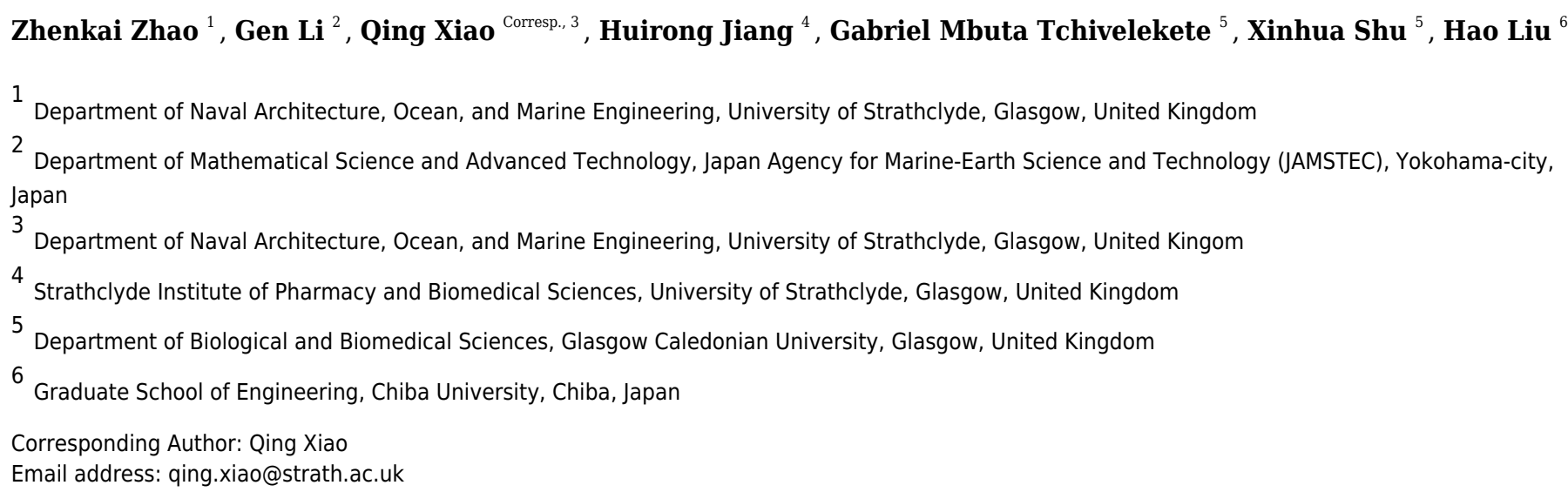

The use of zebrafish larvae has aroused wide interest in the medical field for its potential role in the development of new therapies. The larvae grow extremely quickly and the embryos are nearly transparent which allows easy examination of its internal structures using fluorescent imaging techniques. Medical treatment of zebrafish larvae can directly influence its swimming behaviours. These behaviour changes are related to functional changes of central nervous system (CNS) and transformations of the zebrafish body such as muscle mechanical power and force variation, which cannot be measured directly by pure experiment observation. To quantify the influence of drugs on zebrafish larvae swimming behaviours and energetics, we have developed a novel methodology to exploit intravital changes based on observed zebrafish locomotion. Specifically, by using an inhouse MATLAB code to process the recorded live zebrafish swimming video, the kinematic locomotion equation of a 3D zebrafish larvae was obtained, and a customised Computational Fluid Dynamics (CFD) tool was used to solve the fluid flow around the fish model which was geometrically the same as experimentally tested zebrafish. The developed methodology was firstly verified against experiment, and further applied to quantify the fish internal body force, torque and power consumption associated with a group of normal zebrafish larvae versus those immersed in acetic acid and two neuroactive drugs. As indicated by our results, zebrafish larvae immersed in $0.01 \%$ acetic acid display approximately $30 \%$ higher hydrodynamic power and $10 \%$ higher cost of transport than control group. Besides, 500 uM diphenylhydantoin (DPH) significantly decreases the locomotion activity for approximately 50\% lower hydrodynamic power, whereas $100 \mathrm{mg} / \mathrm{L}$ yohimbine has not caused any significant influences on $5 \mathrm{dpf}$ zebrafish 
larvae locomotion. The approach has potential to evaluate the influence of drugs on the aquatic animal's behaviour changes and thus support the development of new analgesic and neuroactive drugs. 
1 Quantification of the influence of drugs on zebrafish larvae swimming kinematics and energetics 2

3 Zhenkai Zhao ${ }^{1}$, Gen $\mathrm{Li}^{2}$, Qing Xiao ${ }^{1, *}$, Huirong Jiang ${ }^{3}$, Gabriel Mbuta Tchivelekete ${ }^{4}$, Xinhua

$4 \mathrm{Shu}^{4}$ and Hao $\mathrm{Liu}^{5}$

$5 \quad{ }^{1}$ Department of Naval Architecture, Ocean, and Marine Engineering, University of Strathclyde,

6 Glasgow, United Kingdom

7 Email: z.zhao@strath.ac.uk

8 2Department of Mathematical Science and Advanced Technology, Japan Agency for Marine-

9 Earth Science and Technology (JAMSTEC), Yokohama-city, Japan

10 Email: ligen@jamstec.go.jp

11 3Strathclyde Institute of Pharmacy and Biomedical Sciences, University of Strathclyde, Glasgow, 12 United Kingdom

13 Email: Huirong.jiang@strath.ac.uk

$14{ }^{4}$ Department of Biological and Biomedical Sciences, Glasgow Caledonian University, Glasgow,

15 United Kingdom

16 Xinhua.Shu@gcu.ac.uk

$17{ }^{5}$ Graduate School of Engineering, Chiba University, Chiba, Japan

18 Email: hliu@faculty.chiba-u.jp

19 *Author for correspondence (qing.xiao@strath.ac.uk) 


\section{Abstract}

35 The use of zebrafish larvae has aroused wide interest in the medical field for its potential role in the development of new therapies. The larvae grow extremely quickly and the embryos are nearly transparent which allows easy examination of its internal structures using fluorescent imaging techniques. Medical treatment of zebrafish larvae can directly influence its swimming behaviours. These behaviour changes are related to functional changes of central nervous system (CNS) and transformations of the zebrafish body such as muscle mechanical power and force variation, which cannot be measured directly by pure experiment observation. To quantify the influence of drugs on zebrafish larvae swimming behaviours and energetics, we have developed a novel methodology to exploit intravital changes based on observed zebrafish locomotion. Specifically, by using an in-house MATLAB code to process the recorded live zebrafish swimming video, the kinematic locomotion equation of a 3D zebrafish larvae was obtained, and a customised Computational Fluid Dynamics (CFD) tool was used to solve the fluid flow around the fish model which was geometrically the same as experimentally tested zebrafish. The developed methodology was firstly verified against experiment, and further applied to quantify the fish internal body force, torque and power consumption associated with a group of normal zebrafish larvae versus those immersed in acetic acid and two neuroactive drugs. As indicated by our results, zebrafish larvae immersed in $0.01 \%$ acetic acid display approximately $30 \%$ higher hydrodynamic power and 10\% higher cost of transport than control group. Besides, $500 \mu M$ diphenylhydantoin (DPH) significantly decreases the locomotion activity for approximately $50 \%$ lower hydrodynamic power, whereas $100 \mathrm{mg} / \mathrm{L}$ yohimbine has not caused any significant influences on $5 \mathrm{dpf}$ zebrafish larvae locomotion. The approach has potential to evaluate the influence of drugs on the aquatic animal's behaviour changes and thus support the development of new analgesic and neuroactive drugs.

\section{Introduction}

60 In the past decade, the zebrafish has been widely used in medical, biological and genetic

61 research. In its embryonic and larval stage, the zebrafish body is nearly transparent, which 
62 conveniently allows the observation of fish organs development. Its quick reproduction speed 63 and cheaper cost, compared to other fish species and mouse, give it a unique and important role 64 in scientific research to resolve a wide range of issues. Among those issues, nociception and 65

66 nervous system functions are significant and extensively studied. Nociception is a sensory mechanism used to perceive tissue damage (Gregory et al., 2013). Noxious stimuli detected by nociceptors responding to thermal (Malafoglia et al., 2014), electrical (Roques, Abbink, Geurds, van de Vis, \& Flik, 2010) and chemical (Mettam, McCrohan, \& Sneddon, 2012) stimulation can cause acute or chronic pain. zebrafish share similar nociceptive responses to those of human adults (Malafoglia, Bryant, Raffaeli, Giordano, \& Bellipanni, 2013), it is prudent to use the zebrafish larva model to test new analgesic drugs for pain relief. Morphine has been tested on adult zebrafish as an analgesic drug to alleviate the pain caused by acetic acid and shown to have positive effects for pain alleviation. This was achieved via experimental observation and data analysis on fish swimming behaviour changes, such as distance travelled and averaged swimming velocity(Correia, Cunha, Scholze, \& Stevens, 2011; Taylor et al., 2017). Furthermore, more noxious stimuli and drugs have been tested using larvae zebrafish, showing that larvae respond to a noxious challenge in a similar way as adult zebrafish, and the nociceptive response is induced by acetic acid(Lopez-Luna, Al-Jubouri, Al-Nuaimy, \& Sneddon, 2017a), which makes it possible to replace protected adult zebrafish with larvae for nociception research.

Administration of neuroactive drugs is an effective method to test animal's nervous system functions (Irons, MacPhail, Hunter, \& Padilla, 2010).As neuroactive drugs acting on different neural pathways could cause different behavioural phenotypes, it is possible to study how the nervous system affects locomotion behaviours by applying different neuroactive drugs (F. Li et al., 2018). Zebrafish shares similar structure and functions of nervous system compared to mammalian (Anderson \& Ingham, 2003; Xi et al., 2010), and has been validated to study neural effects on behavioural manifestation (Sison, Cawker, Buske, \& Gerlai, 2006). A commonly used drug ethanol was studied to determine the acute and chronic effects on zebrafish behaviours and resulted in different types of behaviour alterations such as time spent active, leaping frequency and distance from stimulus (Dlugos \& Rabin, 2003; R. Gerlai, Lahav, Guo, \& Rosenthal, 2000; Robert Gerlai, Lee, \& Blaser, 2006). Other neuroactive drugs such as cocaine and nicotine were also tested on adult zebrafish and attenuation of swimming activity was observed for both of the 
93 two drugs (Draland \& Dowling, 2001; Levin, Bencan, \& Cerutti, 2007; Lopez-Patino, Yu, 94 Cabral, \& Zhdanova, 2008). Furthermore, zebrafish larvae model was studied with same drugs 95 used in adults and mammalians and showed similar behavioural responses (Irons et al., 2010), 96 suggesting that zebrafish larvae are sensitive to neuroactive drugs.

97 However, observations and quantifications of only distance travelled and velocity are not 98 effective in ascertaining the influence of drugs on fish swimming behaviour. Fish swimming 99 kinematics are controlled by consecutive contractions of muscles located along each side of the

100 body, and the muscle contractions are directly driven by motoneurons in the spinal cord, which is 101 part of the central nervous system (CNS) (Ekeberg, Lansner, \& Grillner, 1995). The bended body 102 controlled by muscle contractions will interact with the surrounding fluid and change its fluid 103 dynamics to power fish swimming (Voesenek, Muijres, \& van Leeuwen, 2018). Under this 104 circumstance, to understand internal muscle mechanics, a useful tool to quantify the association 105 between the effect of drugs on zebrafish swimming behaviour and energetics is required.

106 Although many previous experimental studies managed to visualise fish swimming wake 107 patterns via two-dimensional Particle Image Velocimetry (PIV) flow visualization technique 108 (Muller, 2004; Muller, van den Boogaart, \& van Leeuwen, 2008), this is not sufficient to accurately quantify the influences of the drug on fish swimming, which are mainly reflected via

110 muscle mechanical power and force variation. This is a problem that could be potentially solved

111 with a fully coupled fluid structure interaction (FSI) approach between the fish and the 112 surrounding water.

113

114 Inspired by previous work involving Computational Fluid Dynamics (CFD) simulation on fish 115 swimming(Borazjani \& Sotiropoulos, 2008, 2009; Carling, Willams, \& Bowtell, 1998; J.

116 Lighthill, 1971; Kern \& Koumoutsakos, 2006; G. Li, Muller, van Leeuwen, \& Liu, 2012), a 117 novel nociception-related zebrafish larva model combining a biological methodology and a CFD

118 simulation analysis tool to quantify drug influences on zebrafish locomotion has been developed 119 and described in this paper. Specifically, we used not only observation of live zebrafish 120 swimming behaviour, but also a CFD simulation tool to quantify a number of important 121 swimming characteristics, including body forces and consumption power, which are hard to 122 acquire with experiments only. In this study, we have studied the influence of particular 123 concentration of diphenylhydantoin (DPH), yohimbine and acetic acid on zebrafish larvae 
124 swimming behaviour. The concentration of $500 \mu M \mathrm{DPH}$ and $100 \mathrm{mg} / \mathrm{L}$ yohimbine were selected

125 based on previous research as neuroactive drugs in our study (Q. Li et al., 2015; X. Liu et al.,

126 2016). The concentration of $0.01 \%$ of acetic acid was selected based on Lopez-Luna's

127 experiment setup (Lopez-Luna, Al-Jubouri, Al-Nuaimy, \& Sneddon, 2017b) for a similar

128 pharmacological study including some behavioural studies. In the work of (Peter J. Steenbergen

$129 \&$ Bardine, 2014), they compared the levels of cyclooxygenase-2 (cox-2) in zebrafish larvae and

130 found that activation of nociceptive pathways in a low-concentration acetic acid environment

131 produced behavioural changes that were accompanied by changes in levels of cox- 2 . As the

132 associated gene is involved in nociceptive processes (Bingham, Beswick, Blum, Gray, \&

133 Chessell, 2006), it seems reasonable to say that the acid-induced behavioural changes can be

134 attributed to nociception. Therefore, it is appropriate to use acetic acid for nociception study.

135

136 By comparing the forward swimming speed and hydrodynamic power of wild type zebrafish

137 larvae immersed in water, acetic acid, yohimbine hydrochloride solution and 5, 5-

138 diphenylhydantoin sodium salt solution, we demonstrate that our developed analysis tool is able

139 to quantify some differences, such as fish body internal force and energy consumption, between

140 drugs treated group and control group. As the methodology can, to some extent, quantify the

141 differences of internal muscle mechanics before and after drug treatment, this study has

142 established a foundation for studying the effects of new drugs on zebrafish larvae behaviours.

144 2. Materials and Methodology

145 2.1. Ethics

146 Animal work was carried out in compliance with the Animal Ethics and Welfare Committee,

147 Department of Life Sciences, Glasgow Caledonian University, and UK Home Office under

148 Project License PPL 60/4169.

149

\section{2.2. Experiment setup}

151 The experiment setup (Fig.1A) and main methodologies were developed in our previous study

152 for the determination of the toxicity of acrylamide on zebrafish locomotion via a colour 
153 preference experiment (Jia et al., 2017). In the present study, eighty $5 \mathrm{dpf}$ (days post-

154 fertilization) wildtype zebrafish (Danio rerio) siblings were divided into four groups: the first

155 group (control group) including twenty larvae was immersed in $\mathrm{E} 3$ medium $(5 \mathrm{mM} \mathrm{NaCl}$,

$1560.17 \mathrm{mM} \mathrm{KCl}, 0.33 \mathrm{mM} \mathrm{CaCl}_{2}, 0.22 \mathrm{mM} \mathrm{MgSO}_{4}$, and $0.1 \%$ methylene blue); the second group

157 including twenty larvae was immersed in E3 medium with $0.01 \%$ acetic acid for ten minutes.

158 The third group including twenty larvae was immersed in $500 \mu \mathrm{M}$ diphenylhydantoin (DPH)

159 solution (5,5-diphenylhydantoin sodium salt) and the fourth group including twenty larvae was

160 immersed in $100 \mathrm{mg} / \mathrm{L}$ yohimbine solution (yohimbine hydrochloride). The petri dish was

161 illuminated by a light-emitting diode (LED) panel, driven by an adjustable DC power supply

162 (CSI5003XE, Circuit Specialists, and USA) to provide a continuous and constant light. A high-

163 speed video camera (Mikrotron EoSens CL MC1362) was used to record fish swimming

164 behaviour. The frame rate of the camera was set at 500 frames per second (fps) during the entire

165 experiment process. As in the subsequent CFD numerical modelling, the selected fish with a tail

166 beating frequency being less than $70 \mathrm{~Hz}$, thus, 7-8 frames within one beat cycle is sufficient to

167 capture the fish tail motion. The water temperature was set at as $27^{\circ} \mathrm{C}$ as this is the common

168 temperature widely used in zebrafish experiments. Before the camera started recording, fish in

169 all groups were allowed to swim freely for about 10 minutes to adapt to the water environment;

170 once recording started, there was no stimulation to force the fish swimming forward. In this

171 study, only quasi-steady cruising swimming regime is investigated excluding the sudden-start

172 process. This is evident from previous research that cruising with cyclically motion is essential

173 for fish larvae to cover the distance for migration and dispersal (Sancho, Ma, \& Lobel, 1997). In

174 addition, cruising swimming has been studied extensively, which makes it easier to compare

175 with other researchers' results.

176

177 2.3. Data processing algorithm

178 An in-house MATLAB code was developed and used to post-process the recorded videos and

179 extract zebrafish swimming kinematic characteristics, i.e. motion equations. Figure. 1B depicts

180 the key steps for the process. The original image recorded from the camera was converted to a

181 binary image consisting of the sketch of zebrafish larva only with "im $2 b w$ " function in MATLAB

182 image processing toolbox. With some adjustments and "bwboundaries" function in MATLAB, a 
183 binary image of zebrafish can be extracted, the entire position vector can be obtained for points

184 distributed on fish outline. All images were skeletonized into a single backbone curve using

185 functions "bwmorph" and "thin" operation.

186

187 The coordinated pixels on the backbone curve were then divided into equal-distant segments.

188 These segments were simplified as connected straight lines to calculate relative orientation

189 variation with time between two adjacent segments using MATLAB curve fitting toolbox.

190 Physical representation of the intersection angle is shown in Fig. 1C and the mathematical

191 intersection angle is expressed by Fig. 1D, and calculated with Eqn 1, where $i$ denotes the points

192 numbering from one, and $\theta_{j}$ is the relative angle between each two body segments. As

193 elucidated by (Muller, 2004), the travelling wave of curvature travels along the fish body at a

194 near constant rate, thus an averaged frequency was selected for the entire relative orientation

195 functions. Eqn 2 represents a sample prescribed motion equation for relative angle $\theta_{j}$ between

196 each two body segments.

197

$\theta_{j}=\arctan \left(\frac{y_{i+2}-y_{i+1}}{x_{i+2}-x_{i+1}}\right)-\arctan \left(\frac{y_{i+1}-y_{i}}{x_{i+1}-x_{i}}\right)$

198

$\theta_{j}=a \cos (\omega t)+b \sin (\omega t)$

\subsection{CFD solver \& motion solver}

201

202

203

204

205

206

207

208

209

210

\subsubsection{Zebrafish larva CFD model}

Zebrafish larva model used in OpenFOAM [22] (https://www.openfoam.com/) was built with 51 ellipses extracted from the real fish silhouette and controlled by nine deformation equations as shown in Fig. 2A. To simplify the model, the eyes and fin fold are excluded in the CFD fish model. Density of the fish is assumed to be the same as water. The same average body parameters such as body segments mass and length listed in Table $\mathbf{1}$ is used for all fish in CFD simulation. The flow field was numerically simulated using the open source CFD toolbox OpenFOAM version 3.0.x. The 3-D computational domain is 15 times the fish body length in the longitudinal (x) direction, 10 times of fish body length in transverse (y) direction and 4 times of fish body length in perpendicular $(\mathrm{z})$ direction. The overall fluid domain is assumed to be at rest 
211 initially. In the simulation, the medium is water, therefore, the kinematic viscosity of the fluid $U$,

212 which can be expressed as $\frac{\mu}{\rho}$, is $10^{-6} \mathrm{~m}^{2} / \mathrm{s}$. Pressure boundary conditions are taken as zero

213 gradient for all boundaries except the front and back plane, which are set as symmetry; velocity

214 boundary conditions for fish model were taken as moving wall velocity for all body segments

215 and fixed value for the remaining patches. Local mesh around fish model were depicted in Fig.

216 2B. Mesh around head region before the vertical dash line shown in Fig. 2C is enlarged to be

217 clearer. Considering the constraints in OpenFOAM regarding large mesh deformation to model

218 self-propelled zebrafish swimming, fully unstructured mesh was used to tolerate the internal

219 mesh deformation. For ellipses with high aspect ratio, the mesh is specially refined at the tips to

220 ensure that enough cells are present to precisely capture the vortex at the tips. The Reynolds

221 number is defined as $\frac{v L}{v}, v$ stands for final constant forward swimming velocity, $L$ is the body

222 length of fish larva, and $U$ represents the kinematic viscosity. For the entire simulation, Reynolds

223 number is set as 300, which stands for intermediate flow regime.

\subsubsection{Hydrodynamic solver}

To tackle the CFD mesh motion around zebrafish model, a modified displacementSBRStress motion solver is applied in OpenFOAM. PimpleDyMFoam solver is used to solve the transient, incompressible and single-phase Newtonian fluids. PIMPLE algorithm, a combination of SIMPLE and PISO, is used to address velocity-pressure coupling (Y. Liu, Xiao, Incecik, Peyrard,

$230 \&$ Wan, 2017). Incompressible laminar Navier-Stokes equation was written in Eqn 3 including

231 the conservation equations of mass and momentum. In this equation, U represents the fluid

232 velocity, $p$ is the fluid pressure, $\rho$ is the fluid density, and $U$ is the fluid kinematic viscosity.

233

234

$$
\begin{aligned}
& \nabla \cdot(\mathrm{U})=0 \\
& \underset{\text { timeaccumulation }}{\frac{\partial \mathrm{U}}{\partial \mathrm{U}}}+\underset{\text { convectiontransport }}{\nabla \cdot(\mathrm{U} \mathrm{U})}=-\frac{1}{\rho} \nabla \mathrm{p}+\underset{\text { diffusiontransport }}{\nabla \cdot(\mathrm{v} \nabla \mathrm{U})}
\end{aligned}
$$


236 The time derivatives uses $2^{\text {nd }}$ order implicit discretization scheme, convection term specifies

237 interpolation schemes for velocity as reconCentral, which is different from linear interpolation

238 schemes, it uses extrapolated gradient-based correction from both sides onto the face, using $1 / 2$

239 weighting to increase stability for large deformation.

241 In our case, after receiving position and orientation of the body segments from coupled software,

242 OpenFOAM will interpolate the boundary displacement of fish body to the entire domain to

243 calculate the internal mesh motion. Forces and moments are calculated by integrating the

244 pressure and skin-friction forces over the patches, they calculated forces include pressure forces

245 and viscous forces, which are parallel and perpendicular to the target patch.

\subsubsection{Multibody dynamic software}

The kinematic analysis was based on MBDyn [24], a free general purpose multibody dynamics analysis software developed by the department of aerospace engineering of Polytechnic University of Milan (Politecnico di Milano). It solves initial value problem in the form of Differential-Algebraic Equations (DAE), integrated in time domain using A/L-stable multi-step integration schemes (Y. Li, 2014). Constraints can be added independently in MBDyn, both for rigid and bended body with six degrees of freedom. As our fish model is not a continuous body and is composed of several rigid body segments, it is convenient to use MBDyn to add multiple constraint equations to control the body deformation. By using reference frame, users are able to specify positions, orientations, linear and angular velocities globally and locally. To be specific, calculate the forces and power of fish body by coupling with OpenFOAM. Dynamics of a set of rigid bodies is written in the form of Newton-Euler equations, constrained by Lagrange's multipliers. For unconstrained nodes, the equations of motion are expressed as 
267 Where $\mathrm{x}$ summarizes the $n$ coordinates of the system, $\mathrm{M}(\mathrm{x})$ is the mass matrix, $\mathrm{q}$ summarizes the momentum and momenta moments, and $f$ summarizes the generic force including pressure and viscous force. When the system is subjected to kinematic constraints, the constraints are enforced using Lagrange's multipliers $\lambda$, Differential-Algebraic Equations (DAE) are set as:

271

$272 \mathrm{M}(\mathrm{x}) \dot{\mathrm{x}}=\mathrm{q}$

$273 \dot{\mathrm{q}}+\phi_{/ \mathrm{x}}^{\mathrm{T}} \lambda=\mathrm{f}(\mathrm{x}, \dot{\mathrm{x}}, \mathrm{t})$

$274 \phi(\mathrm{x}, \mathrm{t})=0$

275

276

The Differential-Algebraic Equations are integrated with implicit A/L stable linear multistep

277 integration schemes and a prediction-correction approach is used (Masarati, Morandini, \& Mantegazza, 2014). In our current case, each body segment was expressed as one node in MBDyn. Except the ground node were set as 'static', node, all of the body nodes are dynamic nodes and every two nodes are constrained by a motion equation fitted with MATLAB curve281 fitting toolbox.

282

283

\subsubsection{Coupling strategy}

284 The above two software are coupled using communication primitives provided by MBDyn. To satisfy convergence criteria, a strong coupling, which enables multi-step interactions at each time step, is used between the two software. The schematic diagram for fluid and zebrafish larva motion coupling is shown in Fig. 2D. As indicated in Fig.2D, kinematic data are transmitted bidirectionally. Inter-process communication is built with Transmission Control Protocol (TCP) socket. An external force element in MBDyn allows to communicate positions and orientations of a set of nodes, and the corresponding linear and angular velocities with OpenFOAM, the above data can be transmitted either in global frame or in reference frame. On the other side, once

292 kinematic information is received, the forces and moments are calculated based on the changing 293 positions and orientations and transmitted back to $M B D y n$. Once the convergence criteria are 294 satisfied in OpenFOAM, OpenFOAM stops sending data to MBDyn, and forces and moments in 
295 the latest step would be used by MBDyn and keeps iterating process until convergence. The

296 process is repeated until a final convergent solution is reached.

297

298

\section{Results}

299

\subsection{Validations for multi-body coupling}

300

\subsubsection{Grid independence \& Sensitivity test}

301

A grid independence test was carried out on a self-propelled $0.01 \%$ acetic acid treated zebrafish

302 model with fully prescribed deformation with two mesh sizes, medium and fine mesh. In addition, the time step influence is also tested, e.g. Case 1: Medium mesh (M) with 83200 total cells and time step of T/650, Case 2: Medium mesh \& smaller time step (MS) of 83200 cells and time step of T/1300, and Case 3: Fine mesh (F) of 166400 cells and time step of T/650. The size of the fluid domain remains same for three cases.

To exclude mesh resolution and time step size influences on simulation results, we have compared the forward velocity and total force of zebrafish larvae treated with $0.01 \%$ acetic acid and the results are shown in Fig. 3(A-B). Computational results for three cases including kinematic performance and fluid domain calculation shows close results. To save computational time and keep accuracy, we use the mesh formation and time step size the same as Case1.

312

In addition, we have also tested the sensitivity of the results to body segments of zebrafish larvae model with CFD simulation. Considering the accuracy and efficiency for body segmentation, we

315 have divided the body trunk into 5, 10 and 15 segments, respectively. By capturing the body

316 deformation and simulate the forward motion with CFD toolbox, we compared the forward

317 velocity and total hydrodynamic force in Fig 3(C-D), indicating that the simulation results are

318 not sensitive to the number of body segments.

\subsubsection{Multi-body dynamics validation}

322 To validate our numerical coupling methodology on multi-body structure simulation, a

323 comparison based on jellyfish-inspired swimming provided by Wilson was performed (Wilson \& 
324 Eldredge, 2011). Fig. 4A depicts the jellyfish shape, points on the figure indicate how the main

325 body is separated. Fig. 4B and Fig. 4C illustrate the mathematical model we have created and

326 our CFD model. The prescribed angle functions are represented by $\theta_{1}, \theta_{2}$ and $\theta_{3}$. Detailed

327 functions are expressed with equations (6). By specifying relative orientation equations between

328 every two sections, the jellyfish model can move upwards with alternant contraction and

329 refilling. Different Reynolds numbers were tested, which is defined by the maximum jellyfish

330 diameter and undulation period, i.e. $\frac{\mathrm{D}_{\text {max }}^{2}}{\mathrm{~T} v}, D_{\max }$ is the maximum diameter of the jellyfish model,

$331 T$ is the undulation period, and $V$ denotes kinematic viscosity. Here we selected Re equivalent to

332140 and 70. Fig. 5A and Fig. 5B depict the kinematic performance of the multi-body structure

333 with fully prescribed motion, while Fig. 5C illustrates the power required for the model to move

334 cyclically and Fig. 5D shows the vorticity comparison at $\operatorname{Re}=140$. All of our results are in close

335 consistent with Wilson \& Eldredge's results, the small discrepancies can be caused by the

336 different numerical methods used.

337

338

339

$\theta_{1}=-0.1472 \cos (6.538 t)-0.3247 \sin (6.538 t)+0.7551$

340

$\theta_{2}=-02366 \cos (6.456 t)+0.1645 \sin (6.456 t)+0.3472$

$\theta_{3}=-0.08218 \cos (6.427 t)+0.04095 \sin (6.427 t)+0.4511$

\subsection{Validations on numerical and experimental methodologies}

344 The first objective of our study was to verify the correctness/accuracy and consistence of

345 proposed experiment and CFD method in this work. To achieve this goal, we used the time-

346 varied angle data, extracted via post-processing of experiment video records as input data to

347 CFD fish body discrete elements, the forward motion of fish is the solution by solving a coupled

348 flow solver and multi-body dynamic method as described in Section 2.4. The first and last fish

349 body segment motion is not prescribed whereas they are our numerical solutions, which

350 represent the fish head and tail locomotion. With simulation results, we first compared our

351 yawing head angle and tail beat angle with data collected from observed zebrafish swimming.

352 Fig. 6 (A-B) show an average head and tail angle for 10 individual zebrafish larvae. As seen 
353 from the figures, both the head and tail-beat angles were calculated based on the prescribed 354 constrained deformation equations of zebrafish-matched experimentally observed data. Slight 355 differences can be caused by subtle insufficient accuracy of the fish model capturing toolbox, 356 which might lead to slight inconsistency in certain captured points of head/tail angle. The 357 forward swimming velocity shown in Fig. $\mathbf{6 C}$ reaches about 95\% of the experimentally 358 measured swimming speed, approximately 19.25 body lengths per second. The small gap exists 359 as the real fish may not bend the whole body as symmetrically as our model, which is fully 360 controlled by prescribed sinusoidal functions. The subtle modelling errors may additionally lead 361 to differences in swimming speed. We have also calculated the Strouhal number for simulated

362 CFD results. The Strouhal number $(S t)$ is defined as $\frac{A f}{U}$, where $A$ is the tail beat peak-to-peak

363 amplitude, $f$ is the tail beat frequency and $U$ is the averaged swimming velocity. In our

364 simulation, the Strouhal number is approximately 0.8. A statistical data about forward velocity

365 for 10 zebrafish larvae is shown in Fig. 6D (to save space of the image, only ten results are 366 shown).

Fig. 7 depicts the vorticity iso-surfaces formed based on $Q$ Criterion behind a swimming normal zebrafish larva at different instants in time within one time period and the dorsal view for vorticity iso-surfaces. Here, $Q$ can describe the wake topology and defines vortices as positive second invariant of velocity gradient in region where vorticity magnitude is greater than strainrate magnitude (Koláŕ, 2007). As seen from the most left and right column of Fig. 7, flow patterns behind the fish are represented by detached vortices and shown as translucent green fragments. Vortices starts to form in the vicinity of head, transmits downstream to tail and detaches at the tail, which are consistent with the fish tail motion; when the lateral displacement of the tail reaches the highest amplitude, vortices starts to shed at the tail tip, the already formed vorticity in the wake are mixed with the newly formed vorticity at tail tip. The most right column also displays a 3-D view of the vortex rings generated behind the fish to understand formation of flow patterns better. To validate the numerical methodology, Fig. 7 also compares the body curvatures of CFD model and the real fish in the recorded experiment video. As can be seen, two sets of results match very well in terms of body shape at all specific time within a period, 
382 indicating that our CFD model is able to imitate the self-propelled swimming of zebrafish larva

383 and its interactions with surrounding fluid.

384

385 3.3. Power distribution along fish body and an initial approximation of the cost of 386 transport

387 As the movement of each two neighbouring body segments is constrained with a prescribed

388 deformation equation except for fish head and tail, mechanical power distribution along the fish

389 body can be approximated by power generated by each body segment. The mechanical power

390 generated from fish muscle includes the translational power due to linear motion and the

391 rotational power due to body rotation. As the fish is moving cyclically, all the other terms are

392 cancelled out except for the rotational power. Therefore, the mechanical power is estimated with

393 the cross product of torque and angular velocity shown as equation 7(a), and the total power

394 transmitted into the water is calculated with equation 7(b).

395

396

$397 P_{M}=\sum_{i} M_{i} \cdot \omega_{i}$

398

399

$P_{H}=\sum_{j}-F_{j} \cdot V_{j}$

400

401

$\overline{\mathrm{P}_{\mathrm{M}}}=\overline{\mathrm{P}_{\mathrm{H}}}$

402

403

In the above equation, $\mathrm{P}_{\mathrm{M}}$ is the mechanical power of fish muscle, and $\mathrm{P}_{\mathrm{H}}$ represents

404 hydrodynamic power generated by interactions with surrounding fluid. $M_{i}$ is the internal torque

405 for the $i^{\text {th }}$ joint calculated by MBDyn in the global frame, $\omega_{i}$ represents the angular velocity for

406 the $i^{\text {th }}$ joint. $F_{j}$ is the hydrodynamic force acting on the $j^{\text {th }}$ body and $V_{j}$ represents the $j^{\text {th }}$ body

407 velocity.

408

409

During muscle contraction, fish body bends, energy is generated and transmitted into the water,

410 and the bended body interacts with the surrounding fluid, a thrust force generates and pushes the

411 fish moving forward. Within this process, Approximately $20 \%$ of the energy (depending on fish 
412 species, the percentage can float dramatically) (Zhang, Yu, \& Tong, 2014) generated by muscle

413 contraction is consumed due to the viscous dissipation of fish body tissues, and the remaining

414 energy is transmitted into the water. In our model, viscous dissipations of fish body tissues are

415 neglected to simplify the simulation. Therefore, as the variation of kinetic energy is zero during

416 cyclic swimming, the mechanical power is fully transformed into the hydrodynamic power,

417 which means the absolute value hydrodynamic power equals the mechanical power, and we only

418 need to show hydrodynamic power for 20 zebrafish larvae as depicted in Fig. 8B. The calculated

419 hydrodynamic power has been changed into absolute values as the sign is different from

420 mechanical power. To further understand the different power generated at different locations

421 along the fish body, we have compared the time-history of forces and velocity in Fig. 8 at three

422 typical points shown in Fig. 8A, representing head region, body region and tail region. The

423 trajectory is not completely parallel to $x$ axis in global frame, force and velocity shown in Fig.

424 8C and Fig. 8D are pointing towards the real moving direction of zebrafish. An approximation of

425 hydrodynamic power distribution on each body section is also depicted. As shown in Fig. 8E, the

426 averaged hydrodynamic power for 20 fish larvae shows a significant higher value starting from

427 approximately $75 \%$ of body length. According to motion equations, this region has the largest

428 motion amplitude along the body in global frame, resulting in larger fluid force, thus more

429 hydrodynamic power. In Fig. 8F, the mechanical power generated along the body shows an

430 increase towards the tail and a steep decrease at the tail.

431

432 Detailed mechanical and hydrodynamic power and an approximation of cost of transport are

433 summarised in Table 2. The cost of transport is defined as energy spent to travel unit distance

434 per unit mass, which is expressed as $\frac{P_{m}}{U}, P_{m}$ is the power per unit mass, and $U$ is the forward

435 velocity. Limited by the size of the table, only ten fish data was selected and displayed in the 436 table.

437 3.4. Comparison of kinematics and energetics between normal group and drug treated 438 groups

439 Swimming performance of zebrafish larvae might be affected by drugs with different

440 concentrations. According to Lopez-Luna's research (Lopez-Luna et al., 2017b), zebrafish larvae

441 exposed to $0.01 \%$ acetic acid displayed more active responses than the normal zebrafish, and 
442 these active behaviours were sustained for longer. Liu has tested influences of diphenylhydantoin 443 (DPH) on $5 \mathrm{dpf}$ zebrafish larvae locomotion at different concentrations and found that exposure 444 to higher concentrations of DPH under light condition leads to decreased locomotor activities (X. 445 Liu et al., 2016). Their team also tested the effect of $100 \mathrm{mg} / \mathrm{L}$ yohimbine on $5 \mathrm{dpf}$ zebrafish 446 larvae, but no obvious effect has been found on locomotor activity under light condition (Q. Li et 447 al., 2015). By simulating the swimming behaviours of zebrafish larvae under these circumstance, 448 we examined the forward swimming velocity and cost of transport differences after exposing to $4490.01 \%$ acetic acid, $500 \mu M \mathrm{DPH}$, and $100 \mathrm{mg} / \mathrm{L}$ yohimbine. Fig. 9A and Fig. 9D depict the 450 averaged head and tail comparison for three drugs and control group zebrafish larvae. As the 451 different frequencies and initial angles were used, phase differences existed among those groups 452 in head and tail angle respectively. From the figure, there are no significant differences for the 453 amplitude of head angle, whereas the maximum tail beat angle for zebrafish larva immersed in 454 the $0.01 \%$ acetic acid showed a larger value compared with the rest groups. Fig. 9B illustrates 455 the forward swimming speed comparison between control group zebrafish and drug treated 456 groups. The forward velocity for acetic acid treated zebrafish is dramatically higher than other 457 groups' zebrafish, but there is no significant differences between control group and yohimbine 458 treated group. For zebrafish treated with $500 \mu M$ DPH solution, there is a significant decrease of 459 velocity compared with other groups. These results indicate that the forward swimming velocity 460 might be influenced by the body undulation frequency and tail beat amplitude. With larger

461 frequency and tail beat amplitude being represented by maximum tail beat angle, the forward 462 swimming speed tended to increase.

463

464 As the tail beat frequency and amplitude are both increased, energy generated by fish body will 465 increase as well, which might give rise to lower efficiency as the side oscillations consumes 466 more energy without contributions to thrust. Fig. 9C displays comparisons of the hydrodynamic 467 power $\mathrm{P}_{H}$, cost of transport were calculated for all cases, resulting in $81.73 \mu \mathrm{J} / \mathrm{m} \cdot \mathrm{kg}$, 468 $96.24 \mu \mathrm{J} / \mathrm{m} \cdot \mathrm{kg}, 65.44 \mu \mathrm{J} / \mathrm{m} \cdot \mathrm{kg}$ and $82.32 \mu \mathrm{J} / \mathrm{m} \cdot \mathrm{kg}$ for control, acetic acid treated, DPH 469 treated, and yohimbine treated group, respectively. These values are similar to those reported by $470 \mathrm{Li}$ et al. (from $105 \mu \mathrm{J} / \mathrm{m} \cdot \mathrm{kg}$ to $50 \mu \mathrm{J} / \mathrm{m} \cdot \mathrm{kg}$ in (G. Li, Muller, van Leeuwen, \& Liu, 2016)) on 471 larval zebrafish. It appears that the DPH treated group performed with greater efficiency than the 
472 other groups as it has the lowest tail beat amplitude and frequency. Fig. 9E depicts the forward 473 velocity for all groups. Based on the assumption of no differences among those groups, we have 474 calculated the $P$ value, which is usually evaluated in statistical hypothesis testing to determine 475 the reliability of the results. The $P$ value is smaller than 0.0001 for control group comparison 476 with DPH treated group and acetic acid treated group, which indicates that significant increment 477 of forward velocity can be seen after $0.01 \%$ acetic acid treatment, and a decrease of velocity 478 happens after treated with $500 \mu \mathrm{M}$ DPH solution. There is no obvious differences between 479 control group and yohimbine treated group, indicating that $100 \mathrm{mg} / \mathrm{L}$ concentration of yohimbine 480 will not influence the locomotion behaviour dramatically of $5 \mathrm{dpf}$ zebrafish larvae. Fig. 9F 481 displays similar comparison as Fig. 9E, but with the parameter changed to hydrodynamic power. 482 The resulting $P$ value is smaller than 0.0001 for control group comparison with DPH treated 483 group and acetic acid treated group, leading to the conclusion that averaged hydrodynamic power 484 of acetic acid treated zebrafish larvae is higher than the control group specimens, whereas the 485 zebrafish larvae treated with DPH solution resulted in lower averaged hydrodynamic power. 486 There is no obvious difference between control group and yohimbine treated group as well, 487 which is consistent with results shown in Fig. 9E. Fig. 10 compares the vorticity on x-y plane of 488 the 3-D fish model. By comparing them in one period of time, it can be seen that the vortex 489 detached from the tail tip is faster for acid treated group compared with control group fish. The earlier detached vortex has been labelled with black circles in the right column, indicating the larger distance travelled within one period of time, i.e., higher velocity. For DPH treated group, within same one period of time, the evolution of vorticity is much shorter than other groups, approximately half of other groups, suggesting that only half of the distance travelled by DPH treated group compared with control group. For yohimbine treated group, vorticity patterns are similar to those in control group. All of the vorticity results for drug treated groups are in consistent with velocity comparisons depicted in Fig.9.

\section{Discussions}

499 A novel methodology connecting biological experiments and CFD simulation to explore the 500 relationship between zebrafish larvae fish swimming behaviour and intravital body force/torque 501 changes is proposed and tested in this study. By using the observed zebrafish locomotion, we 
502 extracted the kinematic swimming equations and entered them into our numerical modelling tool

503 to achieve a fluid-body interaction numerical simulation. Although the estimated final cyclic

504 averaged swimming speed of zebrafish larvae via CFD is slightly lower than the experimentally

505 observed results, overall agreement between experiment and CFD is acceptable. Compared with

506 previous studies on zebrafish larvae (G. Li et al., 2012; G. Li et al., 2016), the resultant

507 swimming motion and energetics are within a reasonable range. The estimated Strouhal number

508 is around 0.8 , which is much higher than optimal streamlined fish swimming value of 0.5 , the

509 probable reason might be due to that the zebrafish larvae swims in intermediate flow regime

510 where viscous force dominant, Therefore, overcoming such viscous effect requires more thrust

511 and thus higher Strouhal number is needed (Voesenek et al., 2018). As shown in Fig 7, though

512 we can conclude some differences from the stage of vortex detach by comparing the vorticity in

513 the wake that can be acquired from experimental observations, distinctions between control and

514 acetic treated group are still not clear, which might require quantified data from CFD results

515 described in the previous paragraphs to further identify the differences, indicating the potentials

516 of CFD simulation in the comparisons of nociceptive related studies.

517

518 We have also calculated the hydrodynamic power distribution along the body as shown in Fig

519 5E. Based on our results, the hydrodynamic power generation shows an increase starting from

520 the centre of mass and a steep increase in the rear region. Ideally, the consumption of muscle

521 power requires a study of muscle strain and electromyography (EMG) patterns for muscle

522 function at specific positions along the body; however, the extremely small body size of larval

523 fish makes it impossible to place receivers on the body. Constraints added in our fish model

524 provide energy to move forward from static state, which perform as muscle fibre in real fish to

525 provide mechanical power. Mechanical power distribution has been examined in Fig 5F,

526 showing a steep increase towards the tail from the middle region and then a steep decrease in the

527 tail region. This might suggest that the main power generated by muscle to support steady

528 forward swimming exists in the entire body. The conclusion seems to be inconsistent with the

529 previous viewpoint that most power is generated in the anterior region, while the posterior region

530 performs like a transmitter. However, based on the equations set up in our model, the anterior

531 region equations have smaller curvature, implying that the simulated muscle in this region has

532 smaller strain when it is contracted, thus less positive work is done. Moreover, during steady 
533 swimming state, red muscle dominates the swimming motion; if the main muscle power is

534 generated in the anterior part, loss of energy in the form of heat occurs in the process of force 535 transmission towards the tail, which might increase the burden of red muscle as it powers the 536 entire steady swimming process (Rome, Swank, \& Corda, 1993). Given that muscle functions 537 vary among different species, our results need to be further tested with the help of biological 538 analysis.

539

540

541

542

543

544

545

546

547

548

549

550

551

552

553

554

555

556

557

558

559

560

561

562

563

Acetic acid treated zebrafish might accelerate quicker, i.e., higher frequency, to reach the maximum speed as it attempts to escape the acid environment (Lopez-Luna et al., 2017a). Our results show that the acid treated group achieved higher tail beat frequency and swimming speed than control group. The increment of speed in the intermediate flow regime $\left(10<\operatorname{Re}<10^{3}\right)$ increased the energy dissipation, resulting in higher cost of transport, which agrees with previous study by Li et al. (2012).

Two neuroactive drugs used in this paper, diphenylhydantoin (DPH) and yohimbine are both sensitive to zebrafish larvae, for different days of post fertilization, behavioural changes are different. (Irons et al., 2010). Besides, different concentrations and lighting conditions can cause reversed results for same drug. For example, for $5 \mathrm{dpf}$ zebrafish larvae, $10 \mathrm{mg} / \mathrm{L}$ yohimbine will increase the locomotion activity of fish larvae, whereas $200 \mathrm{mg} / \mathrm{L}$ yohimbine will decrease the activity. In our study, 5 dpf zebrafish larvae applied with $500 \mu M \mathrm{DPH}$ and 100mg/L yohimbine lead to similar results compared with previous biological observations (Q. Li et al., 2015; X. Liu et al., 2016), indicating that our method has the ability to replicate neuroactive drug influences on zebrafish larvae locomotion behaviours. The effect of exposure to acid on zebrafish swimming behaviour has been studied for different substances including acetic acid and citric acid and at different zebrafish developmental stages (Lopez-Luna et al., 2017a; Nordgreen, Tahamtani, Janczak, \& Horsberg, 2014). However, these studies were limited to the nociceptive responses of zebrafish larvae on stress, fear or anxiety, i.e. environment influences. Furthermore, the observed data mainly focused on the total distance fish travelled in a period of time or the time spent in active status(Lopez-Luna et al., 2017a, 2017b). To some extent, our developed tool can mimic the mutual interactions of real fish with the surrounding fluid and thus allows investigation of the relationship between fish body mechanical force and torque and its

Peer) reviewing PDF | (2019:08:40797:2:0:NEW 27 Nov 2019) 
564 swimming behaviours. Using this approach, our future work would focus on evaluating potential

565 analgesic drugs for pain relief and neuroactive drug effects on fish behaviours, which might help

566 to understand functions of nervous system.

567

568 A possible factor that might influence the accuracy of our results is the fish body stiffness, which

569 has not been taken into account in the present research but has been studied by other groups.

570 (McHenry \& van Netten, 2007) Studied the flexural stiffness of superficial neuromasts as it is

571 correlated with the detection of surrounding fluid. Zhang, et al (Zhang, et al,. 2014) provided a

572 prediction of fish body's visco-elastic properties and related muscle mechanical behaviour in

573 vivo based on a continuous beam model (Zhang et al., 2014). Real fish are able to adjust their

574 body stiffness at specific positions in order to optimize their swimming performance such as the

575 maximum forward speed and minimum energy cost (Eric D. Tytell et al., 2016). However, the

576 distribution of visco-elastic properties, i.e. stiffness and damping coefficients along the fish

577 body, are difficult to measure precisely, thus the mutual contributions from visco-elastic

578 properties to the optimized swimming performance cannot be determined individually.

579 Moreover, it is technically difficult to observe subtle body curvature changes. Different fish

580 species may have different stiffness and damping characteristics for different purposes, such as

581 for acceleration/deceleration or cruising swimming (Eric D. Tytell, Hsu, \& Williams, 2010).

582 Considering the importance of body stiffness for a better understanding of muscle functions in

583 controlling fish swimming, we intend to focus in our future research on the visco-elastic

584 properties at some predicted positions with the help of muscle dissection. To be specific, muscle

585 related adverse medical treatment may have effects on muscle tissues such as shortened or

586 dissolved local muscle fibres (Lin, 2012). By applying predicted stiffness and damping

587 coefficients and comparing these with the live fish tissue properties at those locations, it might be

588 possible to account for the influences on altered swimming behaviours.

589

\section{5. Conclusions}

591 In this paper, a novel method has been introduced to quantify the influence of drugs on zebrafish

592 locomotion kinematics and energetics. Experimental results have compared with CFD simulated

593 results on tail beat angle and forwards velocity and showed consistent values. Three types of

594 drugs with positive effect, negative effect and no effect on zebrafish locomotion activity 
595 validated by previous researches have been applied to study applications of our methodology.

596 Reasonable comparison results have been supplied, $0.01 \%$ acetic acid has a positive influence on 5975 dpf zebrafish locomotion, $500 \mu M$ diphenylhydantoin solution has a negative effect on

598 zebrafish locomotion, and the $100 \mathrm{mg} / \mathrm{mL}$ yohimbine will not influence swimming behaviours of

5995 dpf zebrafish larvae significantly. We have also provide results related to internal muscle

600 mechanics, including power distribution along the body and hydrodynamic power comparison,

601 providing insights into internal muscle influences on fish swimming.

602

603 There are still some questions to be solved related to this direction, particularly with respect to

604 how the change of internal muscle could influence swimming behaviours of zebrafish larvae, and

605 how the passive control of fish muscle contributes to swimming efficiency. Evaluation of the

606 analgesic and neuroactive drugs on fish behaviours is also an ongoing effort.

607

608 Acknowledgements

609 This work was supported by laboratory of Department of Biological and Biomedical Sciences in

610 Glasgow Caledonia University. We thank the support from Dr Gen Li and Professor Hao Liu on

611 reviewing the manuscript and apparatus provided by Dr Xinhua Shu for zebrafish experiment.

612

613 Author Disclosure Statement

614 No competing financial interests exist.

615

616

617

618 


\section{References}

Anderson, K. V., \& Ingham, P. W. (2003). The transformation of the model organism: a decade of developmental genetics. Nat Genet, 33 Suppl, 285-293. Retrieved from https://www.ncbi.nlm.nih.gov/pubmed/12610538. doi:10.1038/ng1105

Bingham, S., Beswick, P. J., Blum, D. E., Gray, N. M., \& Chessell, I. P. (2006). The role of the cylooxygenase pathway in nociception and pain. Semin Cell Dev Biol, 17(5), 544-554. Retrieved from http://www.ncbi.nlm.nih.gov/pubmed/17071117. doi:10.1016/j.semcdb.2006.09.001

Borazjani, I., \& Sotiropoulos, F. (2008). Numerical investigation of the hydrodynamics of carangiform swimming in the transitional and inertial flow regimes. The Journal of Experimental Biology, 211(Pt 10), 1541-1558. Retrieved from http://www.ncbi.nlm.nih.gov/pubmed/18456881. doi:10.1242/jeb.015644

Borazjani, I., \& Sotiropoulos, F. (2009). Numerical investigation of the hydrodynamics of anguilliform swimming in the transitional and inertial flow regimes. The Journal of Experimental Biology, 212( $\mathrm{Pt}$ 4), 576-592. Retrieved from http://www.ncbi.nlm.nih.gov/pubmed/19181905. doi:10.1242/jeb.025007

Carling, J., Willams, T. L., \& Bowtell, G. (1998). Self-propelled anguilliform swimming: simulations solution of the two-dimensional navier-stokes equations and newton's laws of motion. The Journal of Experimental Biology, 201, 24.

Correia, A. D., Cunha, S. R., Scholze, M., \& Stevens, E. D. (2011). A Novel Behavioral Fish Model of Nociception for Testing Analgesics. Pharmaceuticals, 4(4), 665-680. doi:10.3390/ph4040665

Dlugos, C. A., \& Rabin, R. A. (2003). Ethanol effects on three strains of zebrafish: model system for genetic investigations. Pharmacology, Biochemistry and Behavior, 74, 10.

Draland, T., \& Dowling, J. E. (2001). Behavioral screening for cocaine sensitivity in mutagenized zebrafish PNAS, 98, 6 .

Ekeberg, O., Lansner, A., \& Grillner, S. (1995). The Neural Control of Fish Swimming

Studied Through Numerical Simulation. Adaptive Behavior, 3.

Gerlai, R., Lahav, M., Guo, S., \& Rosenthal, A. (2000). Drinks like a fish: zebra fish (Danio rerio) as a behavior genetic model to study alcohol effects. Pharmacology, Biochemistry and Behavior, 67, 10.

Gerlai, R., Lee, V., \& Blaser, R. (2006). Effects of acute and chronic ethanol exposure on the behavior of adult zebrafish (Danio rerio). Pharmacology Biochemistry and Behavior, 85, 18.

Gregory, N. S., Harris, A. L., Robinson, C. R., Dougherty, P. M., Fuchs, P. N., \& Sluka, K. A. (2013). An overview of animal models of pain: disease models and outcome measures. J Pain, 14(11), 1255-1269. Retrieved from http://www.ncbi.nlm.nih.gov/pubmed/24035349. doi:10.1016/j.jpain.2013.06.008

Irons, T. D., MacPhail, R. C., Hunter, D. L., \& Padilla, S. (2010). Acute neuroactive drug exposures alter locomotor activity in larval zebrafish. Neurotoxicology and Teratology, 32(1), 84-90. doi:10.1016/j.ntt.2009.04.066

J. Lighthill, M. (1971). Large-Amplitude Elongated-Body Theory of Fish Locomotion (Vol. 179). 
661 Jia, L., Raghupathy, R. K., Albalawi, A., Zhao, Z., Reilly, J., Xiao, Q., \& Shu, X. (2017). A colour 662 preference technique to evaluate acrylamide-induced toxicity in zebrafish. Comp Biochem 663 Physiol C Toxicol Pharmacol, 199, 11-19. Retrieved from 664 http://www.ncbi.nlm.nih.gov/pubmed/28111251. doi:10.1016/j.cbpc.2017.01.004

665

666

667

Kern, S., \& Koumoutsakos, P. (2006). Simulations of optimized anguilliform swimming. The Journal of Experimental Biology, 209(Pt 24), 4841-4857. Retrieved from http://www.ncbi.nlm.nih.gov/pubmed/17142673. doi:10.1242/jeb.02526

668

669

670

671

672

673

674

675

676

677

678

679

680

681

682

683

684

685

686

687

688

689

690

691

692

693

694

695

696

697

698

699

700

701

702

703

704

Kolář, V. (2007). Vortex identification: New requirements and limitations. International Journal of Heat and Fluid Flow, 28(4), 638-652. doi:10.1016/j.ijheatfluidflow.2007.03.004

Levin, E. D., Bencan, Z., \& Cerutti, D. T. (2007). Anxiolytic effects of nicotine in zebrafish. Physiol Behav, 90(1), 54-58. Retrieved from https://www.ncbi.nlm.nih.gov/pubmed/17049956. doi:10.1016/j.physbeh.2006.08.026

Li, F., Lin, J., Liu, X., Li, W., Ding, Y., Zhang, Y., . . Li, Q. (2018). Characterization of the locomotor activities of zebrafish larvae under the influence of various neuroactive drugs. Ann Transl Med, 6(10), 173. Retrieved from https://www.ncbi.nlm.nih.gov/pubmed/29951495. doi:10.21037/atm.2018.04.25

Li, G., Muller, U. K., van Leeuwen, J. L., \& Liu, H. (2012). Body dynamics and hydrodynamics of swimming fish larvae: a computational study. The Journal of Experimental Biology, 215(Pt 22), 4015-4033. Retrieved from http://www.ncbi.nlm.nih.gov/pubmed/23100489. doi:10.1242/jeb.071837

Li, G., Muller, U. K., van Leeuwen, J. L., \& Liu, H. (2016). Fish larvae exploit edge vortices along their dorsal and ventral fin folds to propel themselves. J $R$ Soc Interface, 13(116). Retrieved from http://www.ncbi.nlm.nih.gov/pubmed/27009180. doi:10.1098/rsif.2016.0068

Li, Q., Lin, J., Zhang, Y., Liu, X., Chen, X. Q., Xu, M. Q., . . . Guo, N. (2015). Differential behavioral responses of zebrafish larvae to yohimbine treatment. Psychopharmacology (Berl), 232(1), 197-208. Retrieved from https://www.ncbi.nlm.nih.gov/pubmed/24958231. doi:10.1007/s00213-014-3656-5

Li, Y. (2014). Coupled Computational fluid dynamics/multibody dynamics method with application to wind turbine simulations. Lowa Research online.

Lin, Y. Y. (2012). Muscle diseases in the zebrafish. Neuromuscul Disord, 22(8), 673-684. Retrieved from http://www.ncbi.nlm.nih.gov/pubmed/22647769. doi:10.1016/j.nmd.2012.04.007

Liu, X., Lin, J., Zhang, Y., Peng, X., Guo, N., \& Li, Q. (2016). Effects of diphenylhydantoin on locomotion and thigmotaxis of larval zebrafish. Neurotoxicol Teratol, 53, 41-47. Retrieved from https://www.ncbi.nlm.nih.gov/pubmed/26597863. doi:10.1016/j.ntt.2015.11.008

Liu, Y., Xiao, Q., Incecik, A., Peyrard, C., \& Wan, D. (2017). Establishing a fully coupled CFD analysis tool for floating offshore wind turbines. Renewable Energy, 112, 280-301. doi:10.1016/j.renene.2017.04.052

Lopez-Luna, J., Al-Jubouri, Q., Al-Nuaimy, W., \& Sneddon, L. U. (2017a). Impact of stress, fear and anxiety on the nociceptive responses of larval zebrafish. PLOS one, 12(8), e0181010. Retrieved from http://www.ncbi.nlm.nih.gov/pubmed/28767661. doi:10.1371/journal.pone.0181010

Lopez-Luna, J., Al-Jubouri, Q., Al-Nuaimy, W., \& Sneddon, L. U. (2017b). Reduction in activity by noxious chemical stimulation is ameliorated by immersion in analgesic drugs in zebrafish. 
705

706

707

708

709

710

711

712

713

714

715

716

717

718

719

720

721

722

723

724

725

726

727

728

729

730

731

732

733

734

735

736

737

738

739

740

741

742

743

744

745

746

The Journal of Experimental Biology, 220(Pt 8), 1451-1458. Retrieved from http://www.ncbi.nlm.nih.gov/pubmed/28424313. doi:10.1242/jeb.146969

Lopez-Patino, M. A., Yu, L., Cabral, H., \& Zhdanova, I. V. (2008). Anxiogenic effects of cocaine withdrawal in zebrafish. Physiol Behav, 93(1-2), 160-171. Retrieved from https://www.ncbi.nlm.nih.gov/pubmed/17889042. doi:10.1016/j.physbeh.2007.08.013

Malafoglia, V., Bryant, B., Raffaeli, W., Giordano, A., \& Bellipanni, G. (2013). The zebrafish as a model for nociception studies. J Cell Physiol, 228(10), 1956-1966. Retrieved from http://www.ncbi.nlm.nih.gov/pubmed/23559073. doi:10.1002/jcp.24379

Malafoglia, V., Colasanti, M., Raffaeli, W., Balciunas, D., Giordano, A., \& Bellipanni, G. (2014). Extreme thermal noxious stimuli induce pain responses in zebrafish larvae. J Cell Physiol, 229(3), 300-308. Retrieved from http://www.ncbi.nlm.nih.gov/pubmed/23929528. doi:10.1002/jcp.24447

Masarati, P., Morandini, M., \& Mantegazza, P. (2014). An Efficient Formulation for GeneralPurpose Multibody/Multiphysics Analysis. Journal of Computational and Nonlinear Dynamics, 9(4), 041001. doi:10.1115/1.4025628

McHenry, M. J., \& van Netten, S. M. (2007). The flexural stiffness of superficial neuromasts in the zebrafish (Danio rerio) lateral line. J Exp Biol, 210(Pt 23), 4244-4253. Retrieved from http://www.ncbi.nlm.nih.gov/pubmed/18025022. doi:10.1242/jeb.009290

Mettam, J. J., McCrohan, C. R., \& Sneddon, L. U. (2012). Characterisation of chemosensory trigeminal receptors in the rainbow trout, Oncorhynchus mykiss: responses to chemical irritants and carbon dioxide. J Exp Biol, 215(Pt 4), 685-693. Retrieved from http://www.ncbi.nlm.nih.gov/pubmed/22279076. doi:10.1242/jeb.060350

Muller, U. K. (2004). Swimming of larval zebrafish: ontogeny of body waves and implications for locomotory development. The Journal of Experimental Biology, 207(5), 853-868. doi:10.1242/jeb.00821

Muller, U. K., van den Boogaart, J. G., \& van Leeuwen, J. L. (2008). Flow patterns of larval fish: undulatory swimming in the intermediate flow regime. The Journal of Experimental Biology, 211(Pt 2), 196-205. Retrieved from http://www.ncbi.nlm.nih.gov/pubmed/18165247. doi:10.1242/jeb.005629

Nordgreen, J., Tahamtani, F. M., Janczak, A. M., \& Horsberg, T. E. (2014). Behavioural effects of the commonly used fish anaesthetic tricaine methanesulfonate (MS-222) on zebrafish (Danio rerio) and its relevance for the acetic acid pain test. PLOS one, 9(3), e92116. Retrieved from http://www.ncbi.nlm.nih.gov/pubmed/24658262. doi:10.1371/journal.pone.0092116

Peter J. Steenbergen, \& Bardine, N. (2014). Antinociceptive effects of buprenorphine in zebrafish larvae: An alternative for rodent models to study pain and nociception? Applied Animal Behaviour Science, 152, 92-99.

Rome, L. C., Swank, D., \& Corda, D. (1993). How Fish Power Swimming Science, 261(5119), 4.

Roques, J. A., Abbink, W., Geurds, F., van de Vis, H., \& Flik, G. (2010). Tailfin clipping, a painful procedure: Studies on Nile tilapia and common carp. Physiol Behav, 101(4), 533-540. Retrieved from

http://www.ncbi.nlm.nih.gov/pubmed/20705079. doi:10.1016/j.physbeh.2010.08.001 
747

748

749

750

751

752

753

754

755

756

757

758

759

760

761

762

763

764

765

766

767

768

769

770

771

772

773

774

775

776

777

Sancho, G., Ma, D., \& Lobel, P. (1997). Behavioral observations of an upcurrent reef colonization event by larval surgeonfish Ctenochaetus strigosus (Acanthuridae). Marine Ecology Progress Series, 153, 311-315. doi:10.3354/meps153311

Sison, M., Cawker, J., Buske, C., \& Gerlai, R. (2006). Fishing for genes influencing vertebrate behavior: zebrafish making headway. Lab Animal, 35(5), 33-39. Retrieved from https://doi.org/10.1038/laban0506-33. doi:10.1038/laban0506-33

Taylor, J. C., Dewberry, L. S., Totsch, S. K., Yessick, L. R., DeBerry, J. J., Watts, S. A., \& Sorge, R. E. (2017). A novel zebrafish-based model of nociception. Physiol Behav, 174, 83-88. Retrieved from http://www.ncbi.nlm.nih.gov/pubmed/28288793. doi:10.1016/j.physbeh.2017.03.009

Tytell, E. D., Hsu, C.-Y., \& Williams, T. L. (2010). Interactions between internal forces, body stiffness, and fluid environment in a neuromechanical model of Lamprey swimming. Biophyics and Computational Biology. doi:10.1073/pnas.1011564107/-/DCSupplemental

Tytell, E. D., Leftwich, M. C., Hsu, C.-Y., Griffith, B. E., Cohen, A. H., Smits, A. J., . . Fauci, L. J. (2016). Role of body stiffness in undulatory swimming: Insights from robotic and computational models. Physical Review Fluids, 1(7). doi:10.1103/PhysRevFluids.1.073202

Voesenek, C. J., Muijres, F. T., \& van Leeuwen, J. L. (2018). Biomechanics of swimming in developing larval fish. J Exp Biol, 221(Pt 1). Retrieved from https://www.ncbi.nlm.nih.gov/pubmed/29326114. doi:10.1242/jeb.149583

Wilson, M. M., \& Eldredge, J. D. (2011). Performance improvement through passive mechanics in jellyfish-inspired swimming. International Journal of Non-Linear Mechanics, 46(4), 557567. doi:10.1016/j.ijnonlinmec.2010.12.005

Xi, Y., Ryan, J., Noble, S., Yu, M., Yilbas, A. E., \& Ekker, M. (2010). Impaired dopaminergic neuron development and locomotor function in zebrafish with loss of pink1 function. Eur $J$ Neurosci, 31(4), 623-633. Retrieved from https://www.ncbi.nlm.nih.gov/pubmed/20141529. $\quad$ doi:10.1111/j.14609568.2010.07091.x

Zhang, W., Yu, Y., \& Tong, B. (2014). Prediction of fish body's passive visco-elastic properties and related muscle mechanical performance in vivo during steady swimming. Science China Physics, Mechanics and Astronomy, 57(2), 354-364. doi:10.1007/s11433-013-5372-2 


\section{Figure 1}

Experimental method used to extract zebrafish motion equations

(A) Experiment apparatus for zebrafish swimming video recording. High speed camera is used to capture the fish motion in petri dish (B) zebrafish image extracted from one frame of the video $(C)$ zebrafish outline expressed with white curve and central of mass expressed with green dot (D) zebrafish backbone expressed with white line (E) Equal-distant divisions of the backbone curve, divided with several green dots (F) Expression of relative angle between two segments $(G)$ Intesection angle calculation between each two segments along the backbone.
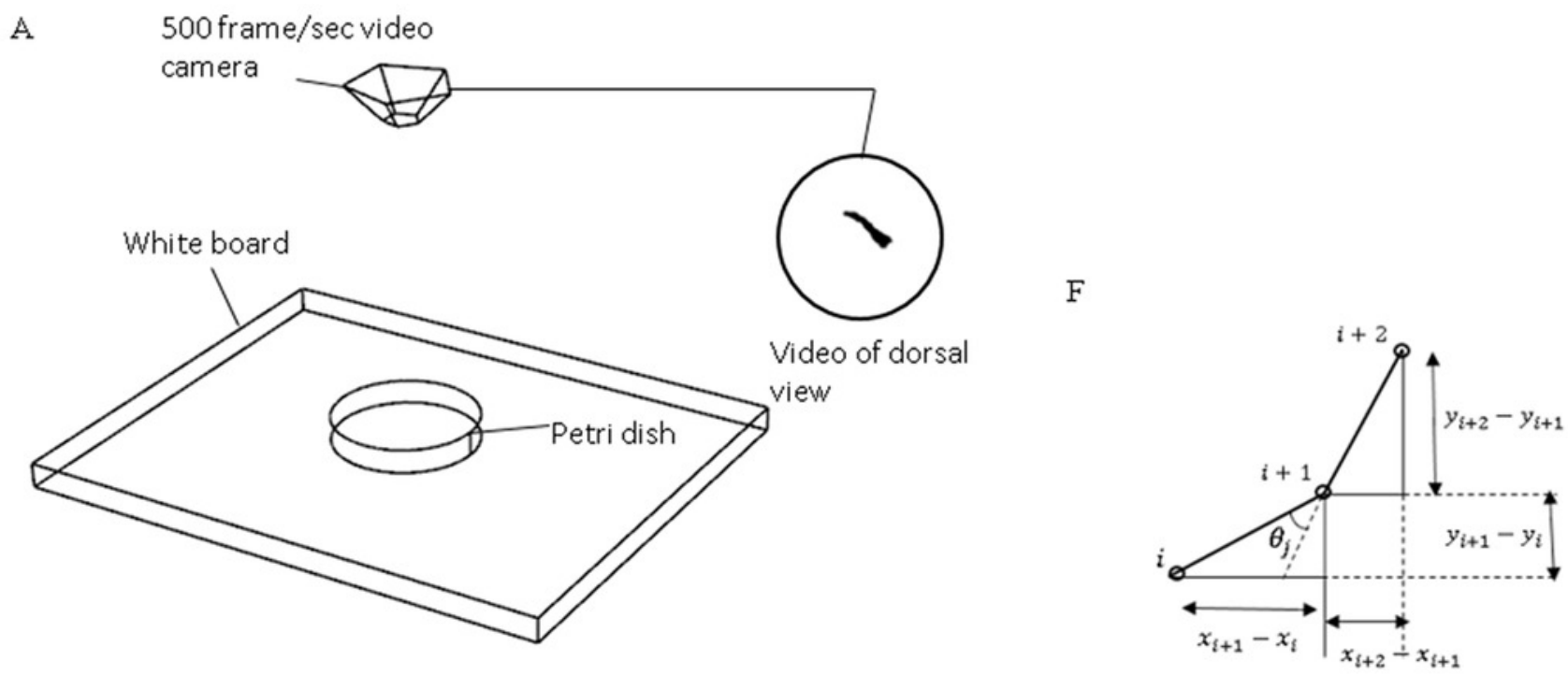

B

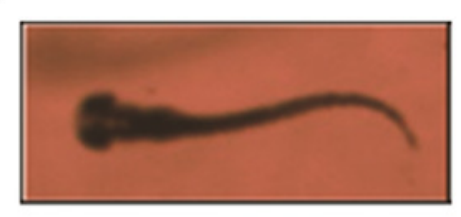

D

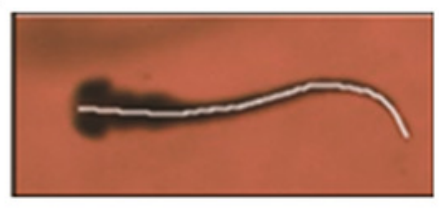

E
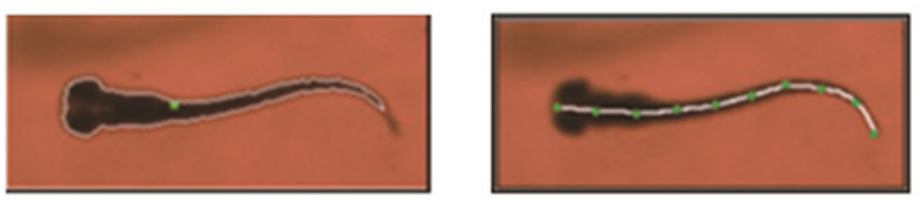

G

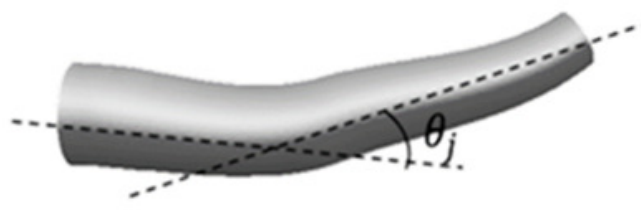




\section{Figure 2}

CFD simulation procedure and mesh geometry of the fish

(A) Flow chart of data transmission between OpenFOAM and MBDyn. Force and displacement vectors are transmitted between OpenFOAM and MBDyn (B) CAD geometry of fish body. The fish body composes of 51 ellipses with different aspect ratio fish body is divided into nine sections with black hollow circle (C) local mesh on fish body in CFD. To accommodate the local mesh rotation and translation, unstructured mesh is built around fish body (D) Enlarged mesh formation around fish head region

A.

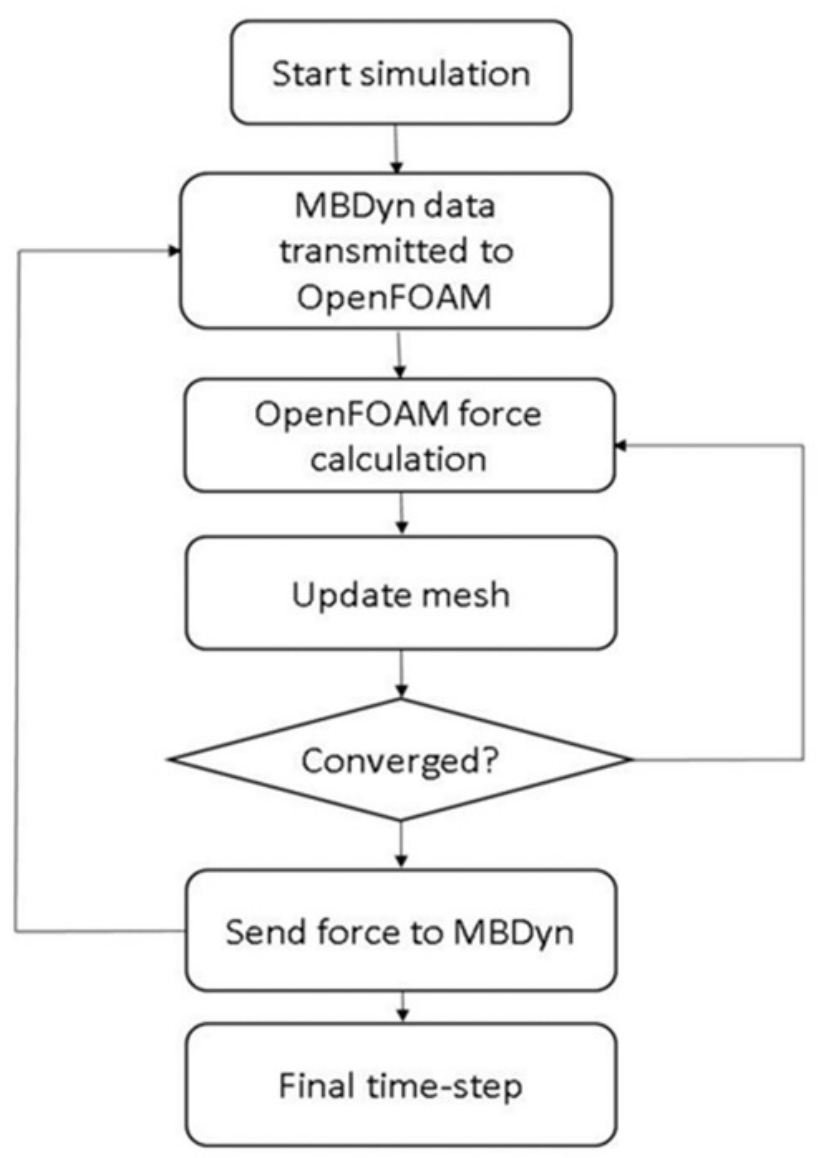

B

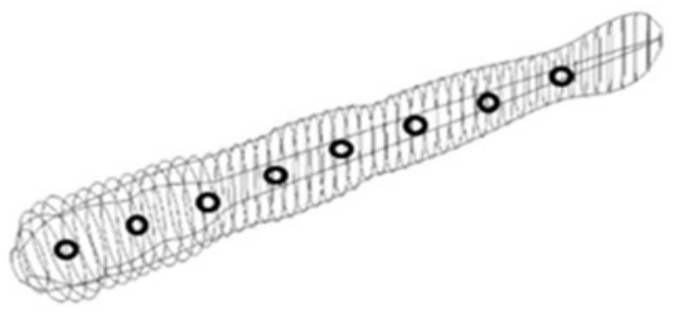

C

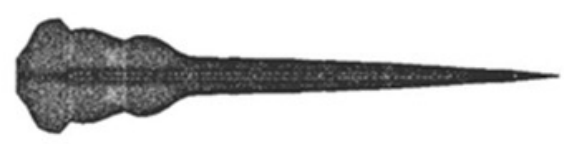

D

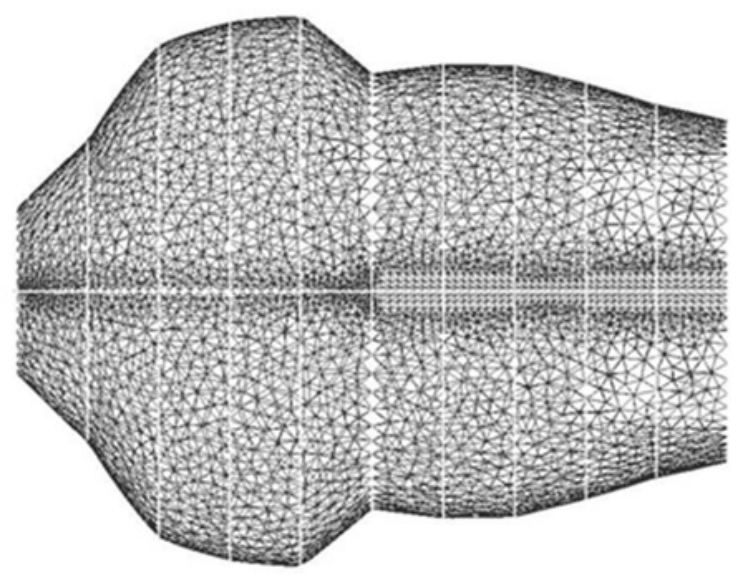


Figure 3

Grid independence test with acetic acid treated zebrafish larva sensitivity study

(A) Forward velocity for three levels of grid. (B) Total force in the moving direction for three levels of grid. (C) Forward velocity for three numbers of segmentations. (D) Total hydrodynamic force for three numbers of segmentations.

A

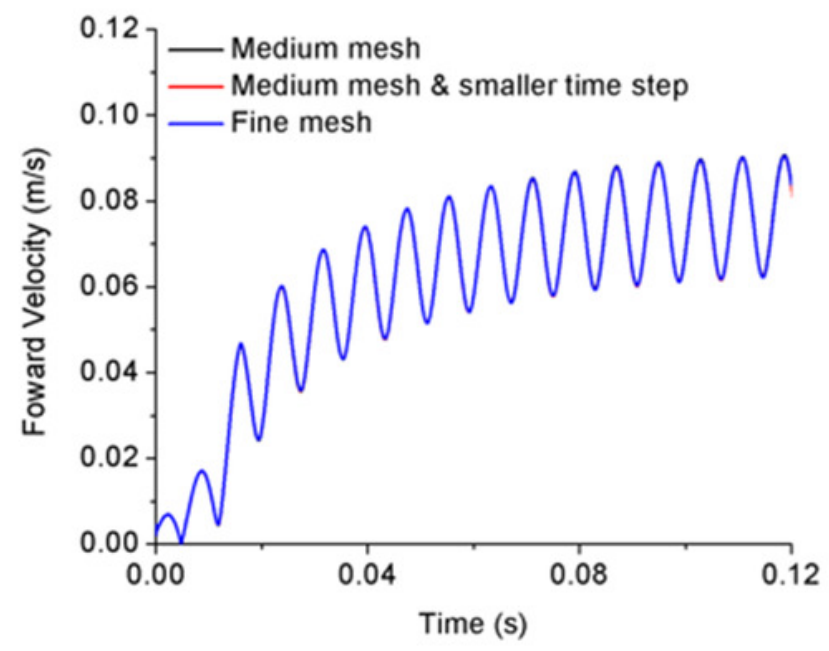

B

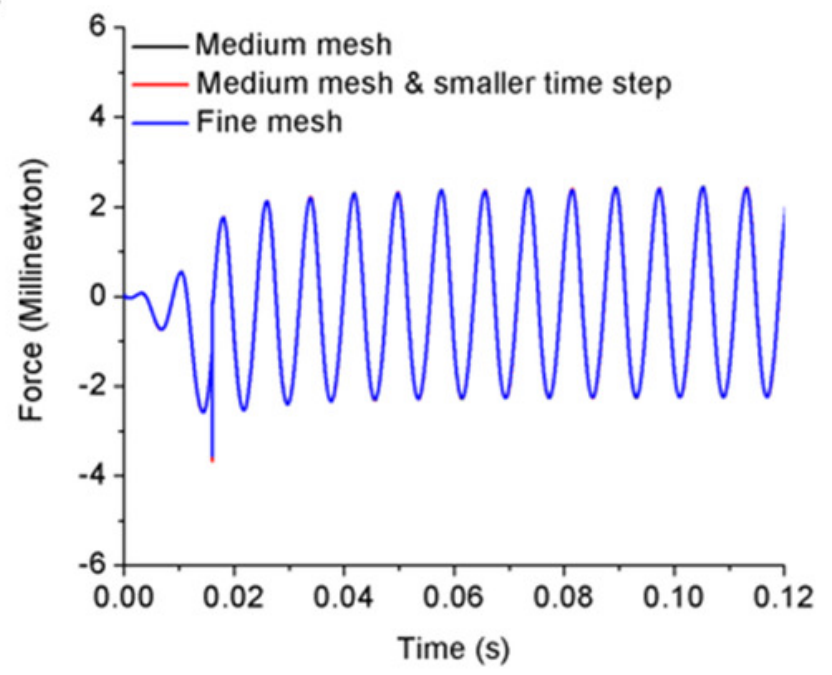

$\mathrm{C}$

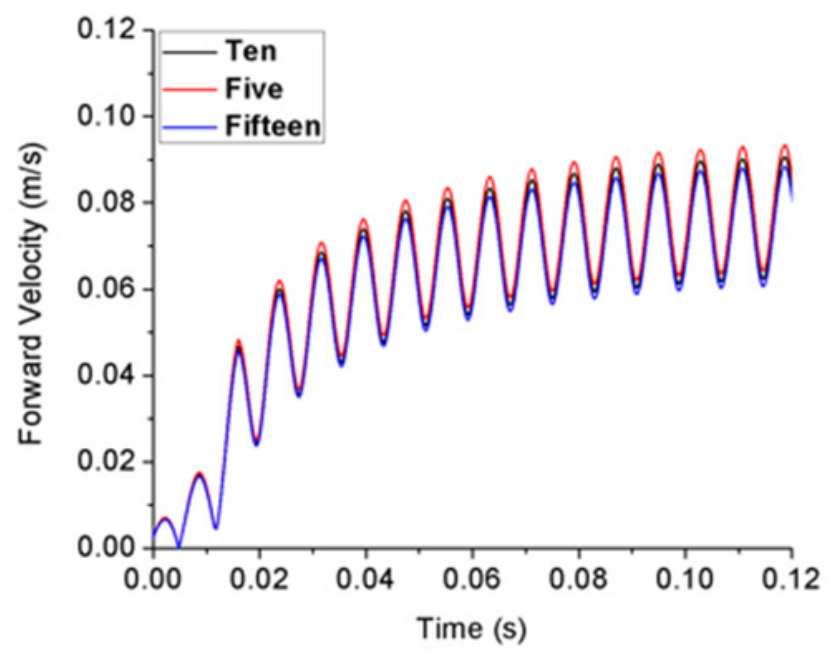

$\mathrm{D}$

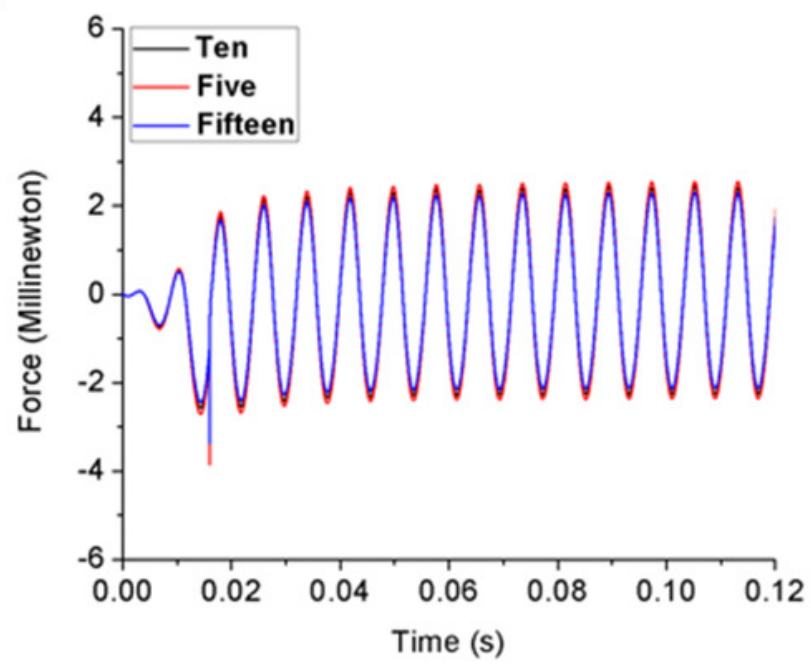


Figure 4

Jellyfish model

Jellyfish model. (A) Real jellyfish shape with dividing points. (B) Mathematical jellyfish model. theta1, theta2 and theta 3 are intersection angles between each two segments. As the structure is symmetric to $Y$ axis, values of intersection angles on the other side is equivalent but with reversed sign. Dmax represents maximum diameter of jellyfish model. (C) Local unstructured mesh around CFD jellyfish model

A

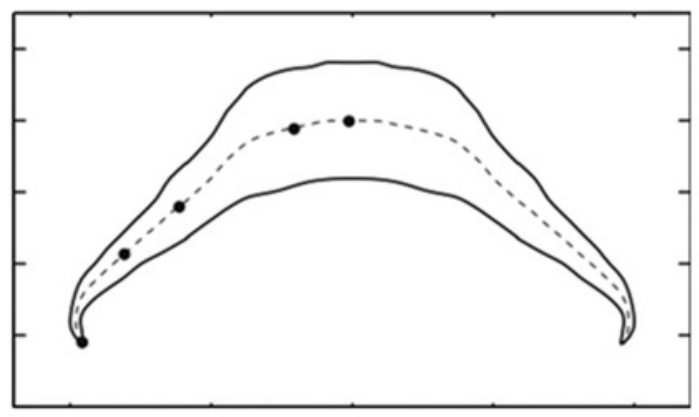

B

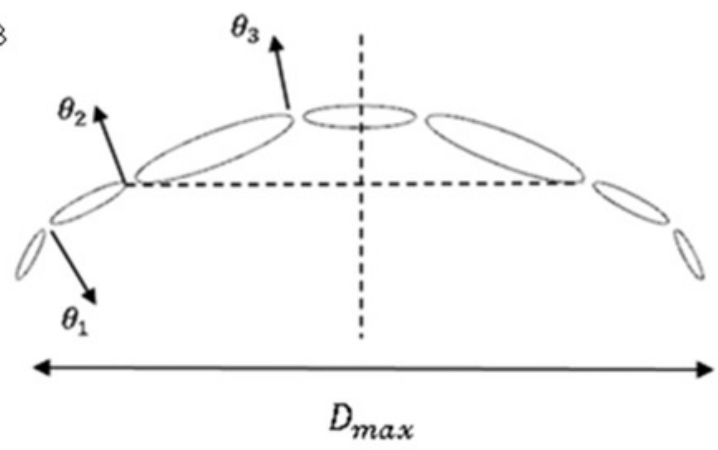

$\mathrm{C}$

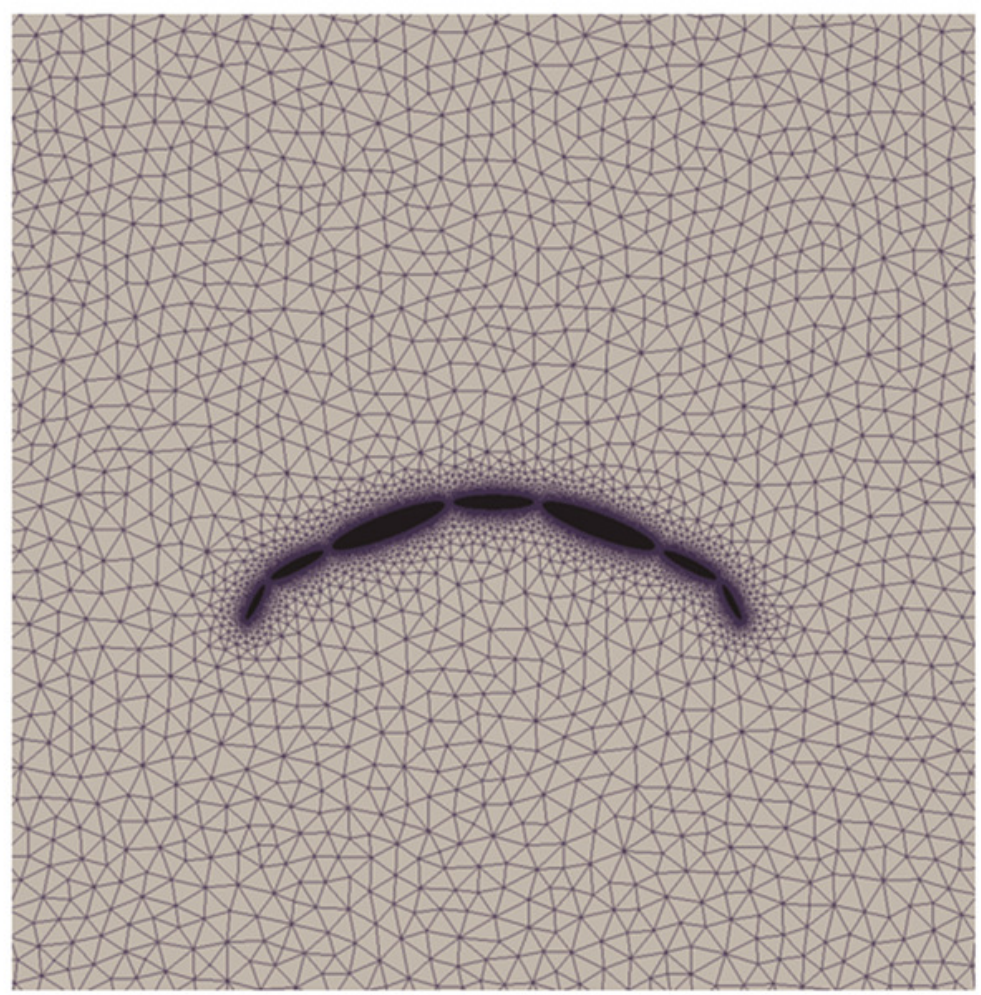


Figure 5

Validation results for periodic jellyfish movements

(A) Longitudinal centroid position for $\mathrm{Re}=140$. (B) Longitudinal centroid velocity for $\mathrm{Re}=$ 140. (C) Required input power for $\operatorname{Re}=70$. (D) Our CFD simulation result of vorticity at $\mathrm{Re}=$ $14($ E) Wilson \& Eldredge's (2011) result of vorticity at $\mathrm{Re}=14$.
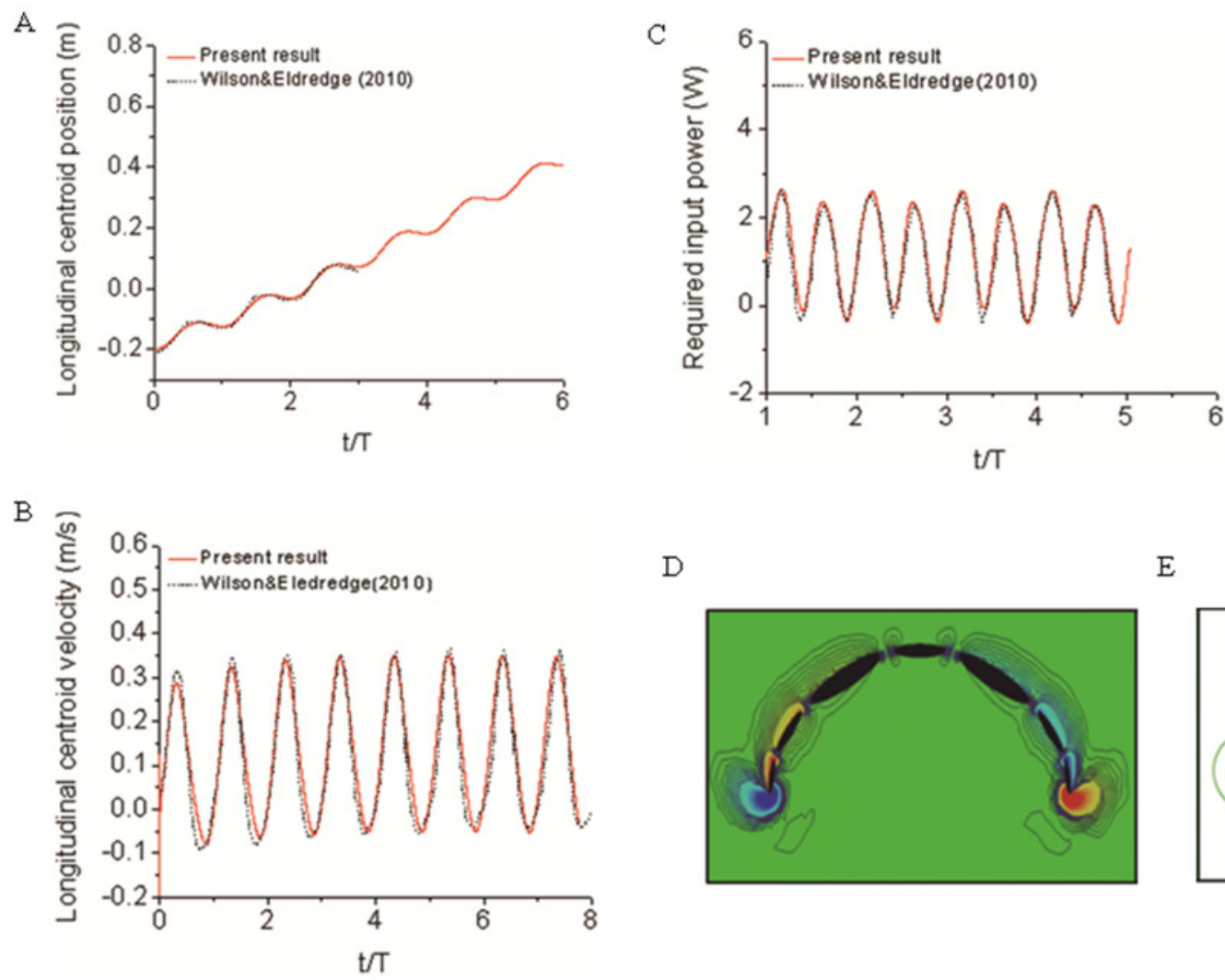

D

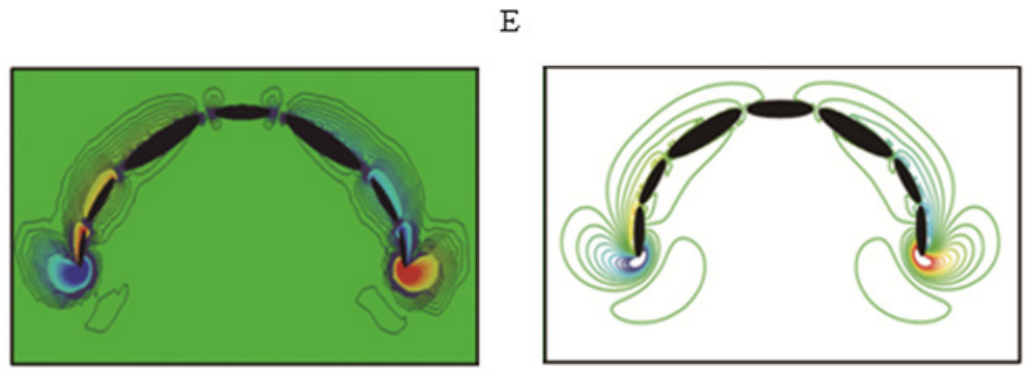


Figure 6

Comparison of simulated zebrafish data in global frame with experimentally observed zebrafish swimming

(A) Head angle (B) Tail angle (C) Forward swimming speed (D) Forward swimming speed for ten fish
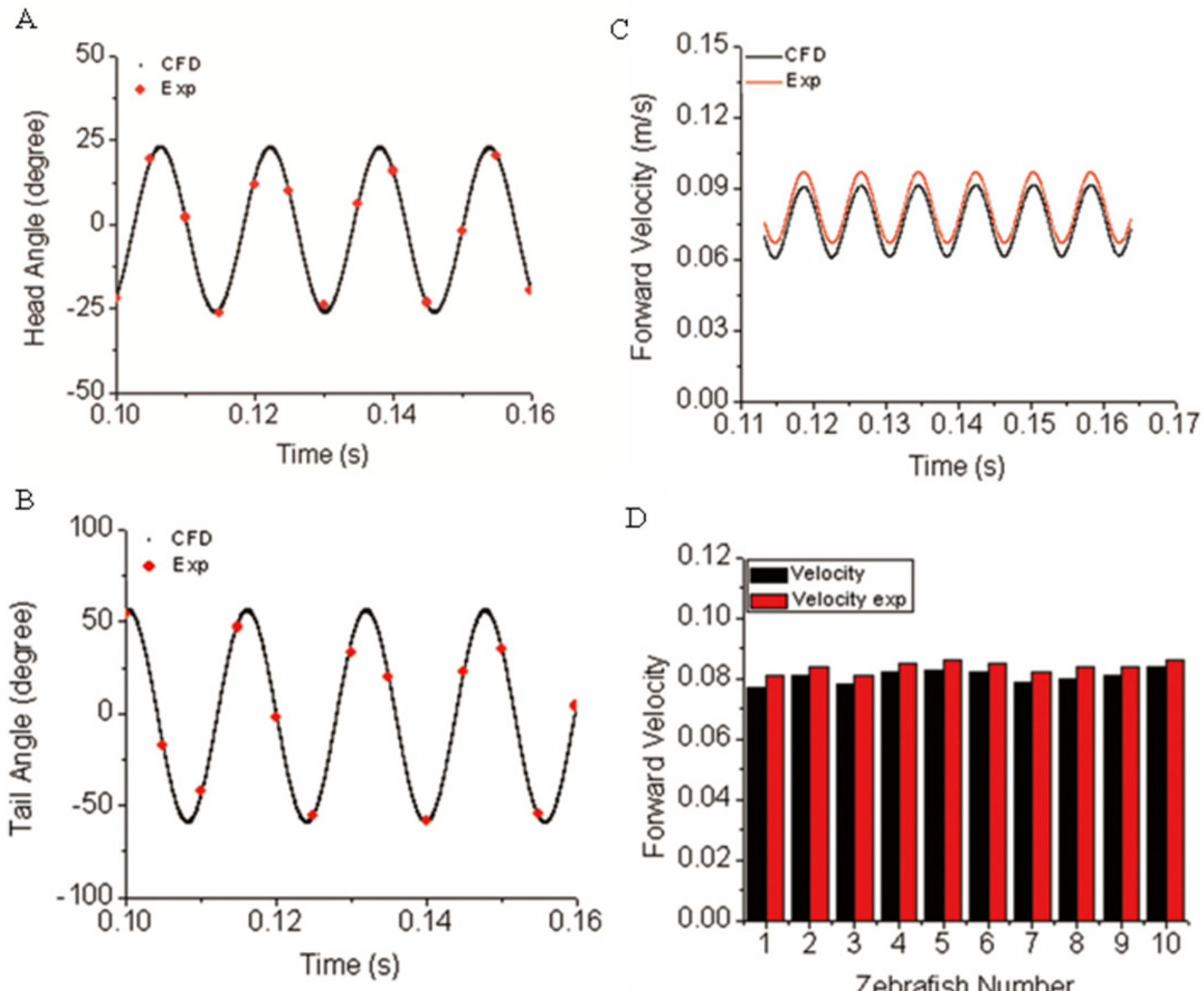

D

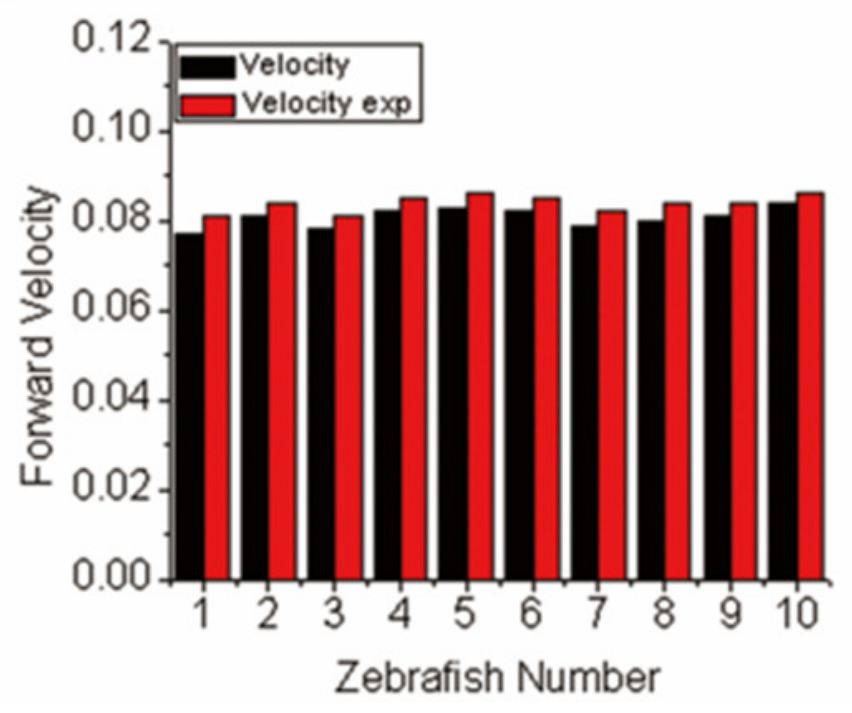


Figure 7

Vortex rings behind zebrafish larva for $\mathrm{Q}=0.5$ at different time step within one period of time and the corresponding video record for the experiment

X-Y plane vorticity is a 2-D view of the 3-D vortices which can compare the body curvature with experiment results easier. From A-D, E-H and I-L, time steps are $0, \mathrm{~T} / 3,2 \mathrm{~T} / 3$ and $\mathrm{T}$ for each column. T represents one period of time.

\begin{tabular}{|l|l|l|}
\hline \multicolumn{1}{|c|}{ X-Y planevorticity } & Experiment & O \\
\hline & & \\
\hline
\end{tabular}


Figure 8

Power distribution at three typical points and along fish body

(A) Real zebrafish picture (B) Hydrodynamic power for ten sample $5 \mathrm{dpf}$ zebrafish larvae (C) Hydrodynamic force at three points (D) Velocity at three points (E) Averaged Hydrodynamic power for twenty fish larvae distribution of each body section along fish body (F) Averaged Mechanical power distribution of each joint along fish body for twenty fish larvae 
A
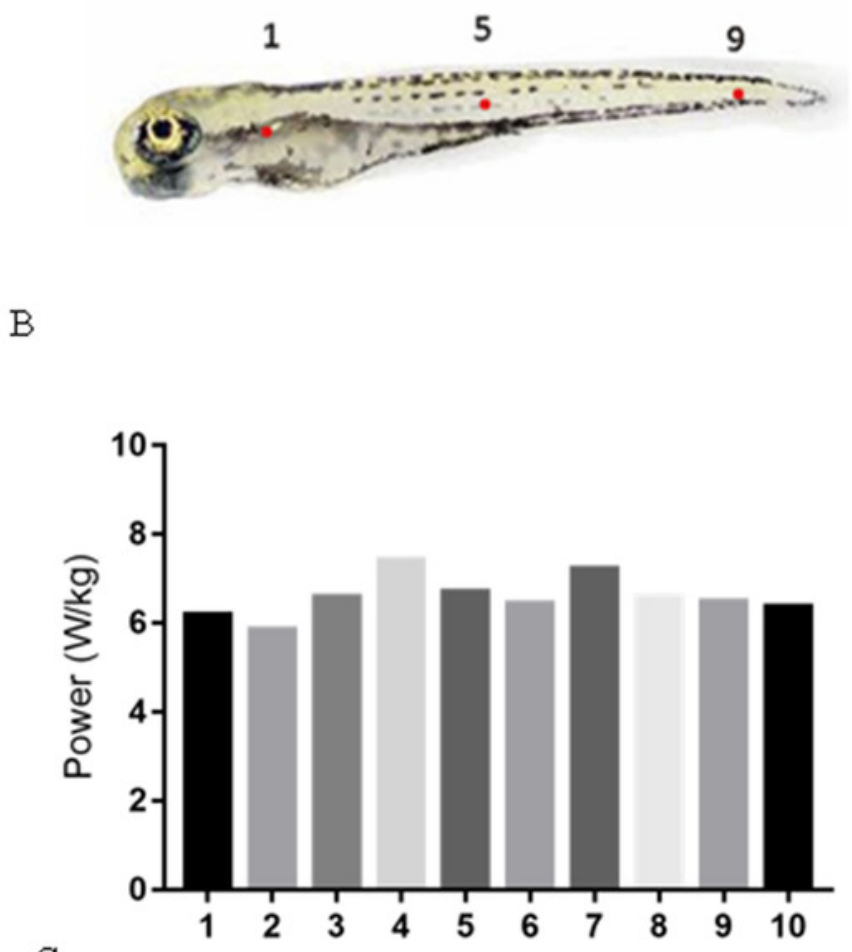

C

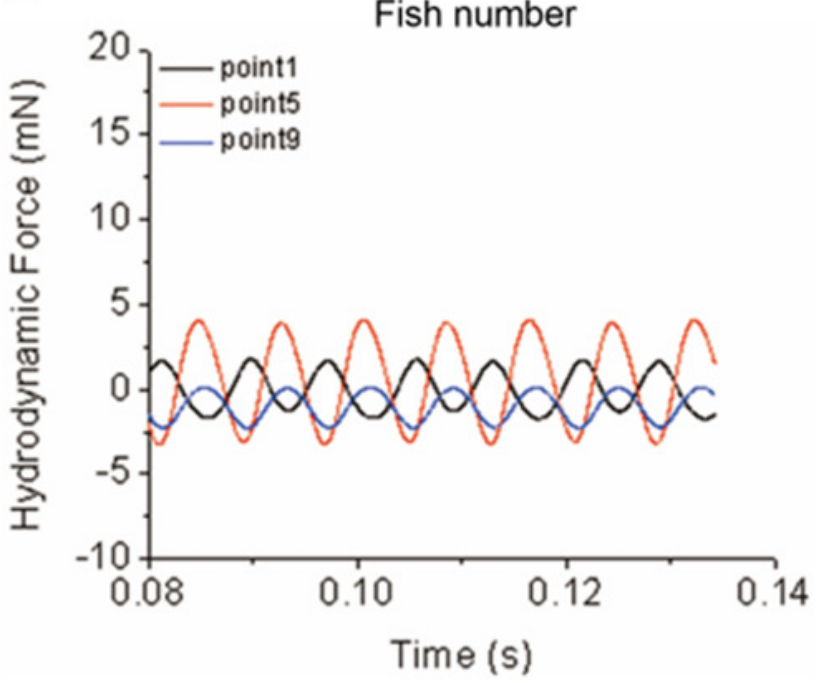

D

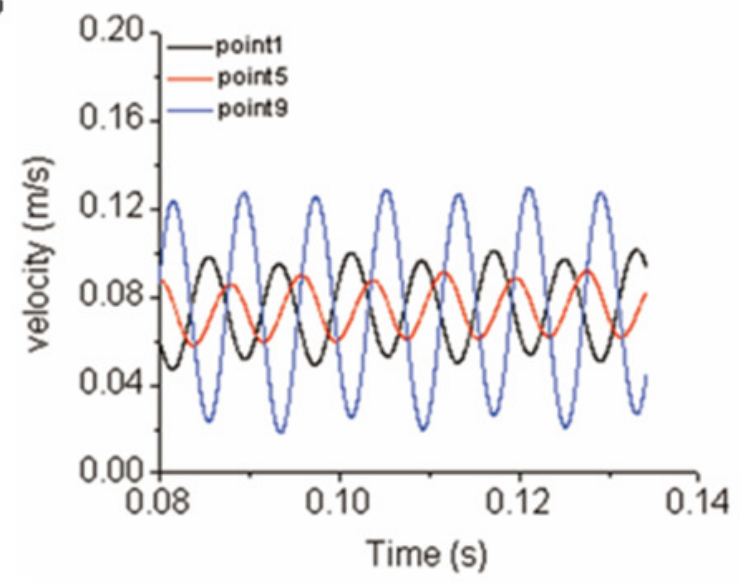

E

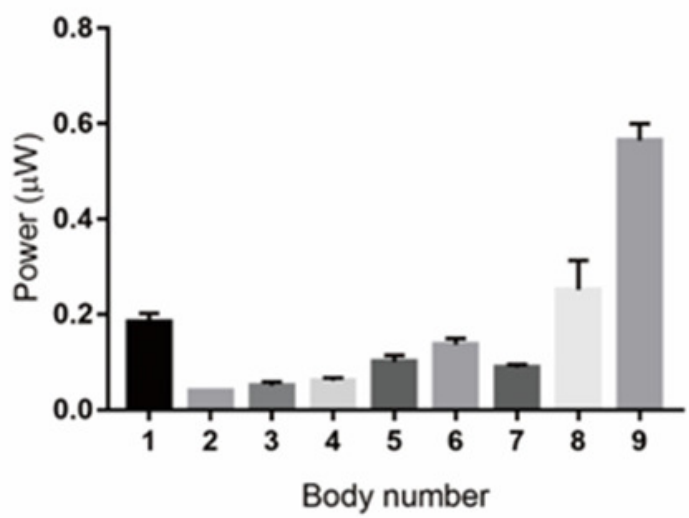

F

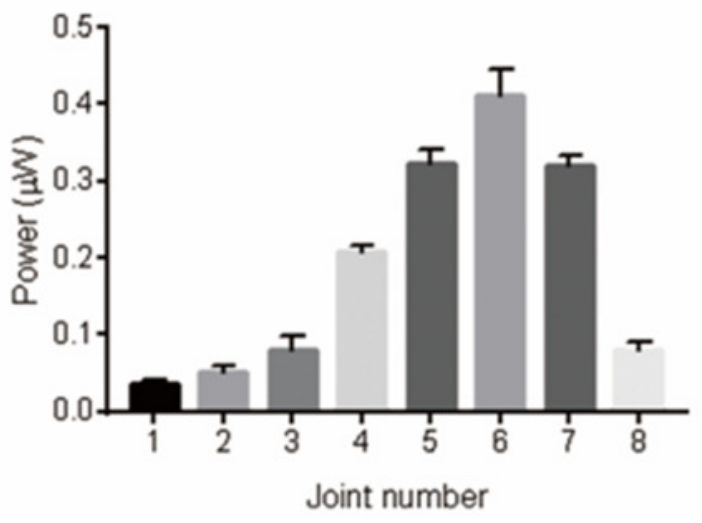




\section{Figure 9}

Comparisons between control group, $0.01 \%$ acetic acid treated group, $500 \mathrm{uM} \mathrm{DPH}$ treated group, and $100 \mathrm{mg} / \mathrm{L}$ yohimbine treated group.

(A) Head angle (B) Forward velocity (C)Hydrodynamic power (D) Tail angle (E) Forward velocity comparison for control group and drug treated groups with two-tailed t-test for twenty fish in all, shown in mean (SD); $\mathrm{P}<0.0001$ : **** (F) Total hydrodynamic power generated by surrounding water with two-tailed t-test for control group and drug treated groups, shown in mean (SD); P $<0.0001$ : ****. 
A
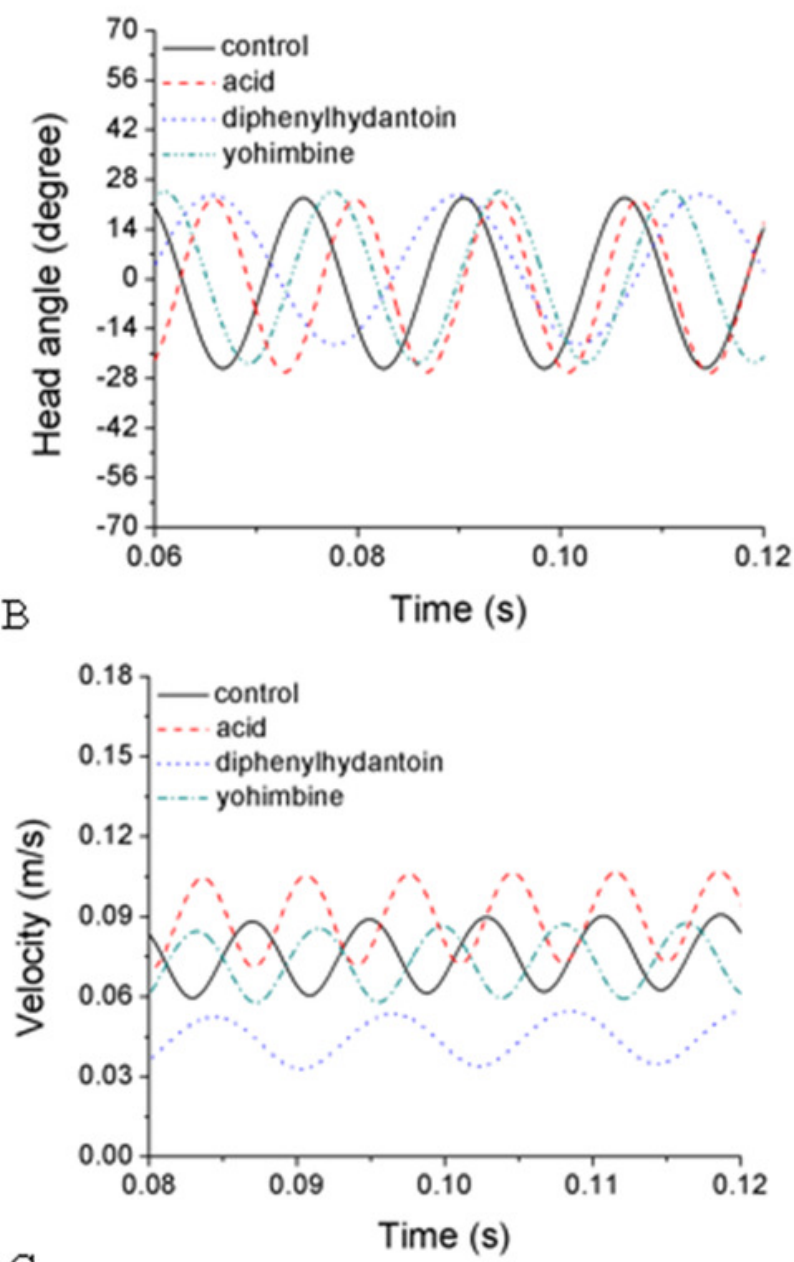

C

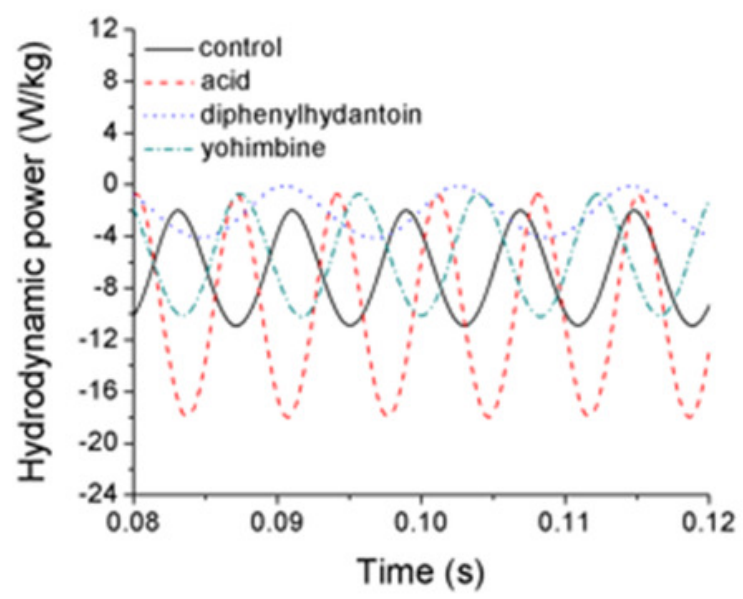

D
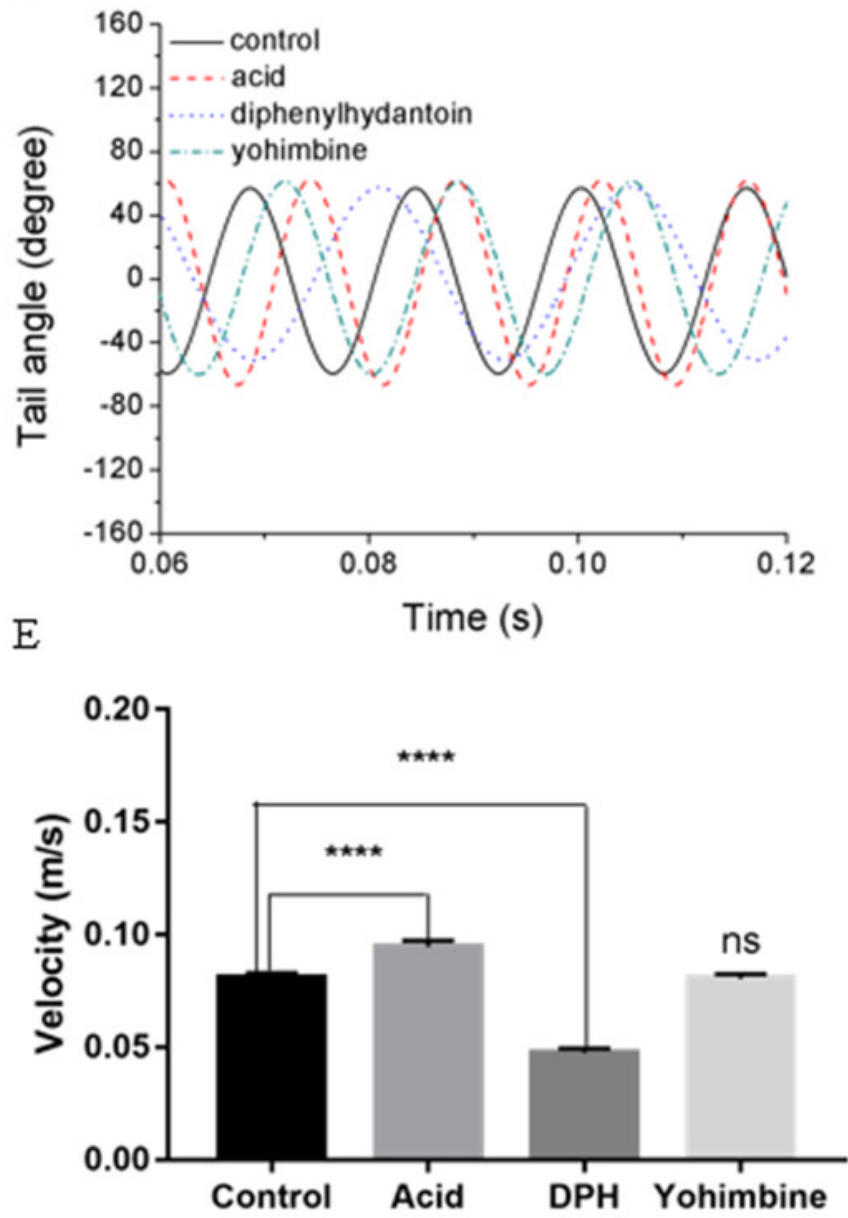

F

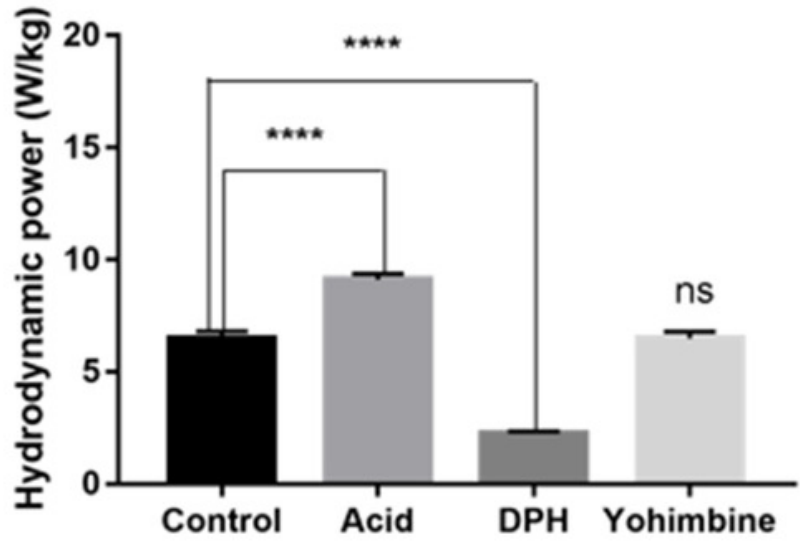




\section{Figure 10}

Vorticity comparisons in $\mathrm{x}-\mathrm{y}$ plane between control group zebrafish and zebrafish exposed to drug treated groups within one period of time

For $\mathrm{A}-\mathrm{D}, \mathrm{E}-\mathrm{H}, \mathrm{I}-\mathrm{L}$ and $\mathrm{M}-\mathrm{P}$, time steps are $0, \mathrm{~T} / 3,2 \mathrm{~T} / 3$ and $\mathrm{T}$ for each column. T represents one period of time.
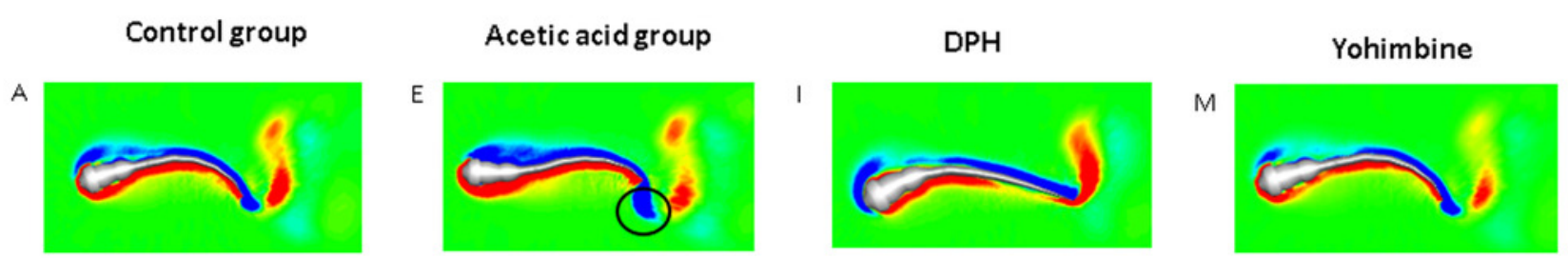

B

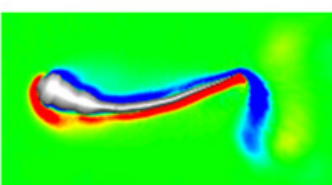

$\mathrm{F}$
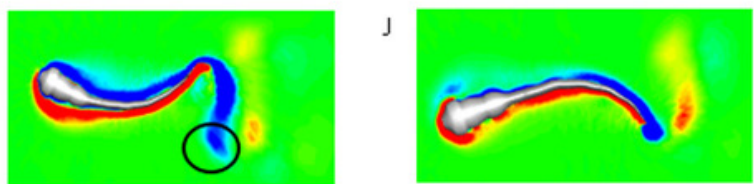

N

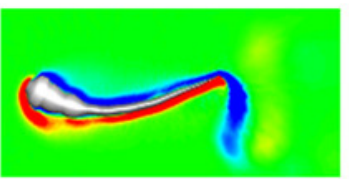

C

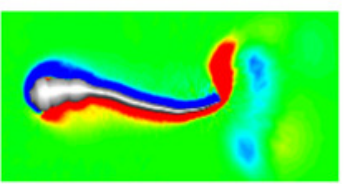

G

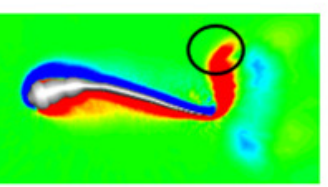

K

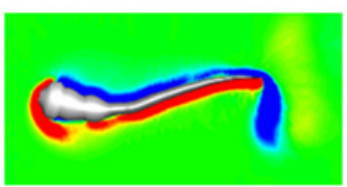

0

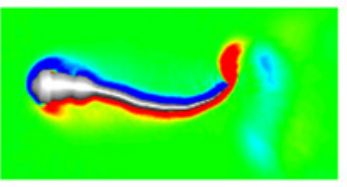

D

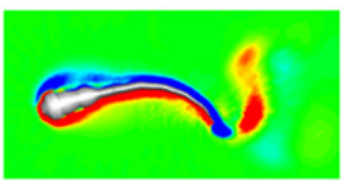

$\mathrm{H}$

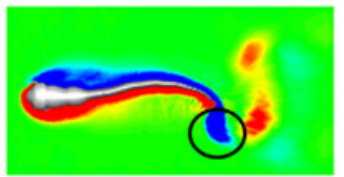

L

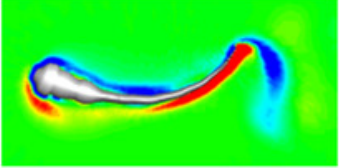

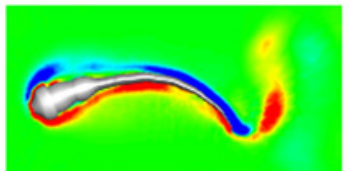


Table $\mathbf{1}$ (on next page)

Detailed zebrafish larva body information 
1

\begin{tabular}{|c|c|c|}
\hline Body section number & Mass $(\mathrm{mg})$ & Length $(\mathrm{mm})$ \\
\hline 1 & 0.0385 & 0.4 \\
\hline 2 & 0.0553 & 0.4 \\
\hline 3 & 0.0425 & 0.48 \\
\hline 4 & 0.0308 & 0.48 \\
\hline 5 & 0.0212 & 0.48 \\
\hline 6 & 0.019 & 0.48 \\
\hline 7 & 0.011 & 0.48 \\
\hline 8 & 0.009 & 0.48 \\
\hline 9 & 0.003 & 0.32 \\
\hline Total & 0.23 & 4 \\
\hline
\end{tabular}

2

3 


\section{Table 2 (on next page)}

Detailed mechanical and hydrodynamic power values for 10 normal zebrafish larva numbered from fish1 to fish 10. S1-S9 represents the nine body sections. Hyd represents hydrodynamic power Mec represents mechanical power units of both are $u W$ 


\begin{tabular}{|c|c|c|c|c|c|c|c|c|c|c|c|}
\hline & & S1 & S2 & S3 & S4 & S5 & S6 & S7 & S8 & S9 & $\begin{array}{c}\text { cost of } \\
\text { transport } \\
\mu \mathrm{J} / \mathrm{m}\end{array}$ \\
\hline \multirow[t]{2}{*}{ fish1 } & Hyd & 0.16 & 0.04 & 0.05 & 0.06 & 0.09 & 0.14 & 0.08 & 0.26 & 0.55 & \multirow[t]{2}{*}{ 79. 97} \\
\hline & $\mathrm{Mec}$ & 0.00 & 0.03 & 0.04 & 0.06 & 0.21 & 0.32 & 0.38 & 0.31 & 0.07 & \\
\hline \multirow[t]{2}{*}{ fish2 } & Hyd & 0.17 & 0.04 & 0.03 & 0.05 & 0.08 & 0.12 & 0.08 & 0.26 & 0.51 & \multirow[t]{2}{*}{78.78} \\
\hline & $\mathrm{Mec}$ & 0.00 & 0.03 & 0.04 & 0.06 & 0.20 & 0.31 & 0.36 & 0.30 & 0.07 & \\
\hline \multirow[t]{2}{*}{ fish3 } & Hyd & 0.19 & 0.04 & 0.06 & 0.07 & 0.11 & 0.14 & 0.09 & 0.26 & 0.56 & \multirow[t]{2}{*}{84.33} \\
\hline & $\mathrm{Mec}$ & 0.00 & 0.03 & 0.05 & 0.09 & 0.21 & 0.32 & 0.41 & 0.33 & 0.08 & \\
\hline \multirow[t]{2}{*}{ fish4 } & Hyd & 0.23 & 0.04 & 0.05 & 0.06 & 0.12 & 0.15 & 0.10 & 0.08 & 0.63 & \multirow[t]{2}{*}{90.57} \\
\hline & Mec & 0.00 & 0.04 & 0.06 & 0.12 & 0.22 & 0.37 & 0.49 & 0.34 & 0.08 & \\
\hline \multirow[t]{2}{*}{ fish5 } & Hyd & 0.18 & 0.04 & 0.05 & 0.07 & 0.12 & 0.16 & 0.09 & 0.28 & 0.55 & \multirow[t]{2}{*}{80.64} \\
\hline & Mec & 0.00 & 0.04 & 0.06 & 0.09 & 0.21 & 0.31 & 0.42 & 0.33 & 0.08 & \\
\hline \multirow[t]{2}{*}{ fish6 } & Hyd & 0.19 & 0.04 & 0.05 & 0.06 & 0.08 & 0.14 & 0.09 & 0.28 & 0.54 & \multirow[t]{2}{*}{77.61} \\
\hline & $\mathrm{Mec}$ & 0.00 & 0.03 & 0.05 & 0.06 & 0.20 & 0.33 & 0.40 & 0.31 & 0.08 & \\
\hline \multirow[t]{2}{*}{ fish7 } & Hyd & 0.18 & 0.04 & 0.06 & 0.06 & 0.10 & 0.14 & 0.09 & 0.28 & 0.59 & \multirow[t]{2}{*}{85.56} \\
\hline & $\mathrm{Mec}$ & 0.00 & 0.04 & 0.06 & 0.09 & 0.22 & 0.31 & 0.42 & 0.33 & 0.10 & \\
\hline \multirow[t]{2}{*}{ fish8 } & Hyd & 0.18 & 0.04 & 0.05 & 0.06 & 0.10 & 0.13 & 0.09 & 0.27 & 0.55 & \multirow[t]{2}{*}{79.66} \\
\hline & $\mathrm{Mec}$ & 0.00 & 0.04 & 0.04 & 0.07 & 0.20 & 0.32 & 0.41 & 0.31 & 0.08 & \\
\hline \multirow[t]{2}{*}{ fish9 } & Hyd & 0.18 & 0.04 & 0.05 & 0.06 & 0.10 & 0.13 & 0.09 & 0.27 & 0.55 & \multirow[t]{2}{*}{ 79. 18} \\
\hline & Mec & 0.00 & 0.03 & 0.05 & 0.06 & 0.21 & 0.32 & 0.42 & 0.30 & 0.08 & \\
\hline \multirow[t]{2}{*}{ fish10 } & Hyd & 0.18 & 0.04 & 0.05 & 0.06 & 0.10 & 0.12 & 0.09 & 0.28 & 0.61 & \multirow[t]{2}{*}{81.01} \\
\hline & Mec & 0.00 & 0.04 & 0.06 & 0.09 & 0.21 & 0.31 & 0.41 & 0.33 & 0.09 & \\
\hline
\end{tabular}

